\title{
A Three-Dimensional Gas-Kinetic BGK Scheme for Simulating Flows in Rotating Machinery
}

\author{
Di Zhou, Zhiliang Lu* and Tongqing Guo
}

Department of Aerodynamics, College of Aerospace Engineering, Nanjing University of Aeronautics and Astronautics, Qinhuai District, Nanjing 210016, Jiangsu, China

Received 31 March 2018; Accepted (in revised version) 18 May 2018

\begin{abstract}
This paper focuses on the development and application of a threedimensional gas-kinetic Bhatnagar-Gross-Krook (BGK) method for the viscous flows in rotating machinery. For such flows, a rotating frame of reference is usually used in formulating the Navier-Stokes (N-S) equations, and there are two major concerns in constructing the corresponding BGK model. One is the change of the convective velocities in the N-S equations, which can be reflected through modification of the gas streaming velocity. The other one is the necessity to account for the effect of the additional Coriolis and centrifugal forces. Here, a specifically-designed acceleration term is added into the modified Boltzmann equation so that the source effects can be naturally included into the gas evolution process and the resulted fluxes. Under the finitevolume framework, the constructed BGK model is locally solved at each cell interface and then the numerical fluxes can be evaluated. When employing the BGK scheme, it is sometimes found that the calculated spatial derivatives of the initial and equilibrium distribution functions are sensitive to the mesh quality especially in complex rotating flow applications, which may significantly influence flux evaluation. Therefore, an improved approach for computing these slopes is adopted, through which the modeling capability for viscous flows is enhanced. For validation, several numerical examples are presented. The computed results show that the present method can be well applied to a wide range of flows in rotating machinery with favorable accuracy.
\end{abstract}

AMS subject classifications: $76 \mathrm{U} 05$

Key words: Gas-kinetic scheme, BGK model, non-inertial reference frame, acceleration term, rotating machinery.

\section{Introduction}

The study of flows in rotating machinery has been an attractive and challenging issue. Examples of such flows cover a wide range of scientific and engineering applications [1],

*Corresponding author.

Email: luzl@nuaa.edu.cn (Z. L. Lu) 
such as rotating Couette flow, rotating cavity flow, and turbomachinery flow. There are significant differences between rotating and non-rotating fluid applications. The first one is the presence of centrifugal and Coriolis forces in a rotating flow, which could be responsible for the possible differences in dynamics and aerodynamic performance. Secondly, the complex secondary flow pattern appearing in rotating machinery can be another distinction. It is caused by the combined effects of boundary layer formation, wake interactions and rotation. For example, many types of secondary flows occur in turbomachinery, including tip leakage, passage vortex, and corner vortex. Besides, in some cases the effect of flow rotation may lead to complex toroidal vortices and even flow instabilities. A notable example is the Taylor-Couette flow, where axisymmetric and non-axisymmetric instabilities appear when the characteristic angular velocity is increased above certain thresholds. These distinguishing features all increase the difficulty of accurate modeling of flows in rotating machinery and thus set a higher demand for numerical methods.

Currently, most computational fluid dynamics (CFD) computations are based on solving Navier-Stokes equations. Under the finite-volume framework, the inviscid fluxes are usually evaluated using upwind schemes, while the viscous fluxes are evaluated employing central differences. Typical upwind schemes include flux difference splitting (FDS)-type and flux vector splitting (FVS)-type schemes, which are both derived from the discretization of the Euler equations. However, practice has shown that the computational accuracy and robustness may vary depending on the selected numerical scheme, especially in boundary layer and shock structure calculations. Furthermore, numerical instabilities are sometimes encountered, such as the carbuncle phenomena and odd-even decoupling. These difficulties may be further enhanced in simulating rotating flows, probably causing significant impact to performance calculation and prediction of secondary flow pattern. Generally, we can attribute them to the excessive or insufficient numerical dissipation, which is implicitly provided in the scheme but does not meet the requirement in various flow regions. There are, however, more physical reasons to explain this. It is demonstrated in [2] that due to the difference between physical and numerical fluid, the Euler equations may not be an appropriate physical model to describe the time evolution of numerical fluid in all situations. Also because most upwind schemes use quasi-one-dimensional approaches, they may show quite different behaviors in multidimensional cases [3].

In the recent decade, there has been a growing interest in constructing schemes based on the Boltzmann equation since it has a more fundamental physical basis. The gaskinetic BGK scheme (BGK), which was first proposed by K. H. Prendergast and K. Xu [4], is probably one of the most popular Boltzmann-type methods. Because of its many merits such as the multidimensionality, the delicate dissipative mechanism and the positive property, the BGK scheme can give accurate and robust N-S solutions. Particularly in the discontinuous regions, it has proven to be capable of capturing a crisp and stable shock structure [5]. With efforts of scholars, many useful BGK schemes have been developed for a wide variety of flows, as reported in [6-10]. As for rotating fluid applications concerned in this work, it also shows great potential in improving flow simulation. 
It is an usual way to express the governing N-S equations in a rotating frame of reference. Because the rotating system is not inertial, additional centrifugal and Coriolis forces exist, serving as macroscopic source terms. In conventional N-S solvers, the source effects are usually neglected in the evaluation of numerical fluxes. As a contrast, in BGK schemes, they should be accounted for in the construction of the BGK model, because only with an appropriate gas evolution model can the concerned N-S equations be recovered. This is also a necessary basis of the present method. Since the numerical fluxes at the cell interface are obtained by reconstructing the solution of the BGK model, the source effects are naturally included in the resulted fluxes. Note that the importance of including source terms into the gas evolution process has also been demonstrated by $\mathrm{Xu}$ [11] in developing a BGK scheme for heat transfer problems. Recently, for simulating two-dimensional viscous flows around arbitrarily moving bodies, the authors developed a moving reference frame-based BGK scheme (MRF-BGK) [12], where the non-inertial effects are considered by adding a particle acceleration term into the Boltzmann equation. With the Chapman-Enskog expansion analysis, it was proven that the developed BGK model correctly recovers the N-S equations in a general moving frame of reference. Here, we follow the same idea and extend it for three-dimensional rotating flows.

Accurate modeling of viscous effects is extremely crucial to rotating flows, because there are usually several secondary flows superposed on the mainstream flow and any deviation in "predicted" viscous forces may lead to entirely different flow patterns. As analyzed in [13], the viscous part of fluxes in the BGK scheme is contributed by a nonlinear average of spatial derivatives of the initial non-equilibrium state and the equilibrium state. Therefore, the gradients of flow variables should be obtained both at the cell interface and in left and right cells. Note that many scholars [5, 13-15] employ a more general way to approximate the equilibrium state so that the gradients on either side of the cell interface are required. Originally they were all calculated using a simple difference approximation, and there are two consequences of this. First, it may be inefficient to deal with non-Cartesian mesh, thus a coordinate transformation approach [16] or a modified approach proposed in [13] have to be employed. Secondly, it is found that this way sometimes leads to unphysical results and even numerical instabilities. The reason is that the computed gradients could be strongly sensitive to the mesh quality, or more specifically, the distance between the cell centers and the distance between the cell center and the cell interface. This problem can be more severe in rotating fluid applications such as in turbomachinery, because the computational domain is usually a narrow and warped passage where several highly distorted meshes exist. To remove this problem and meanwhile maintain the multidimensionality of the BGK scheme, an efficient approach for computing the derivatives of the initial and equilibrium states is adopted. It is also worth mentioning that $\mathrm{Xu}$ et al. [17] and his collaborators [18,19] did a lot of work for the development of an intrinsically multidimensional BGK scheme, where the basic idea is to include both normal and tangential gradients in the flux evaluation.

This paper is organized as follows. First we introduce the construction of the BGK model for the N-S equations in a rotating reference frame. Then the solving process for 
the developed BGK model is presented in detail. In particular, the calculations of the spatial derivatives of initial and equilibrium states by using an improved approach are addressed. Finally, several numerical examples are used for validation.

\section{Methodology}

\subsection{Construction of the BGK model for the N-S equations in a rotating reference frame}

Owing to the unsteady nature of rotating flows as seen by a stationary observer, it is a convenient way to use a rotating frame of reference, through which the flow motion can be thought as predominantly steady relative to the rotating bodies. The angular velocities are assumed to have a general expression, i.e., $\boldsymbol{\omega}=\omega \mathbf{r}_{r o t}$, where $\mathbf{r}_{r o t}=\left[r_{x}, r_{y}, r_{z}\right]^{T}$ denotes a unit vector in the direction of the rotation axis. Due to the reasons explained in [20], it is preferred to use the absolute velocity formulation to express both the macroscopic equations and the Boltzmann equation. Since the present BGK scheme is designed to solve the N-S equations formulated in a rotating reference frame, they are first given as

$$
\begin{aligned}
& \frac{\partial \rho}{\partial t}+\nabla \cdot\left(\rho \mathbf{v}_{r}\right)=0, \\
& \frac{\partial \rho \mathbf{v}}{\partial t}+\nabla \cdot\left(\rho \mathbf{v} \mathbf{v}_{r}+p \mathbf{I}\right)-\nabla \cdot \overline{\overline{\boldsymbol{\tau}}}=-\rho(\boldsymbol{\omega} \times \mathbf{v}), \\
& \frac{\partial \rho E}{\partial t}+\nabla \cdot\left(\rho E \mathbf{v}_{r}+p \mathbf{v}\right)-\nabla \cdot(k \nabla T+\overline{\overline{\boldsymbol{\tau}}} \cdot \mathbf{v})=0,
\end{aligned}
$$

where $\rho, p, T, E$, and $k$ are the density, pressure, temperature, total energy per unit mass and thermal conductivity coefficient, respectively. The absolute velocity vector $\mathbf{v}$ and the relative velocity vector $\mathbf{v}_{r}$ have the components $\mathbf{v}=[u, v, w]^{T}$ and $\mathbf{v}_{r}=\left[u_{r}, v_{r}, w_{r}\right]^{T}$, I is the identity matrix, and $\overline{\overline{\boldsymbol{\tau}}}$ is the viscous stress tensor. It is noted that in the momentum equations, the Coriolis and centrifugal forces have been simplified into a single term, acting as an external source.

By using the finite volume method, an integral form of Eq. (2.1) for a particular volume $\Omega$ gives

$$
\Omega \frac{d \mathbf{W}}{d t}=-\sum_{m=1}^{N_{F}}(\mathbf{F} \Delta S)_{m}+\Omega \mathbf{Q}
$$

where $N_{F}=6$ for structured mesh, $\Delta S_{m}$ is the area of face $m, \mathbf{W}=[\rho, \rho \mathbf{v}, \rho E]^{T}$ is the conservative variables defined at the cell center, $\mathbf{F}_{m}$ represents the total fluxes defined at the cell interface, and $\mathbf{Q}$ is the macroscopic source term.

As compared with the original BGK model, there are two major concerns in constructing the BGK model for the above macroscopic equations. The first one is to modify the gas streaming velocity in the Boltzmann equation since the convective velocities have 
changed from $\mathbf{v}$ to $\mathbf{v}_{r}$ as shown in Eq. (2.1). The other one is to account for the source effects in the constructed BGK model. Here, by introducing a particle acceleration term into the modified Boltzmann equation, we have

$$
\frac{\partial f}{\partial t}+\xi^{\prime} \cdot \nabla f+\mathbf{a}_{\xi} \cdot \nabla_{\xi} f=-\frac{1}{\tau}(f-g),
$$

where $f$ is the gas distribution function, $g$ is the equilibrium distribution function, $\tau$ is the particle collision time, $\xi^{\prime}=\left[\xi_{x}{ }^{\prime}, \xi_{y}{ }^{\prime}, \xi_{z}{ }^{\prime}\right]^{T}$ is a vector of the modified streaming velocities, and $\mathbf{a}_{\xi}=\left[a_{\xi, x}, a_{\xi, y}, a_{\xi, z}\right]^{T}$ is a vector of the particle accelerations. The notation $\nabla_{\xi}$ represents the gradient of a scalar with respect to the vector of particle velocities $\xi_{z}=\left[\xi_{x}, \xi_{y}, \xi_{z}\right]^{T}$. The streaming velocities $\xi_{x}{ }^{\prime}, \xi_{y}{ }^{\prime}$ and $\xi_{z}{ }^{\prime}$ are respectively defined as $\xi_{x}{ }^{\prime}=\xi_{x}-u_{e}, \xi_{y}{ }^{\prime}=\xi_{y}-v_{e}$ and $\xi_{z}{ }^{\prime}=\xi_{z}-w_{e}$, and $u_{e}, v_{e}$ and $w_{e}$ are components of the vector of entrainment velocities $\mathbf{v}_{e}=\mathbf{v}-\mathbf{v}_{r}$. The particle accelerations $a_{\xi, x}, a_{\xi, y}$ and $a_{\xi, z}$ will be determined later. Both $f$ and $g$ are functions of space $x=(x, y, z)$, time $t$, particle velocities $\left(\xi_{x}, \xi_{y}, \xi_{z}\right)$ and internal variables $\zeta_{i},(i=1,2, \cdots, N)$. The equilibrium state $g$ is usually defined as a Maxwellian

$$
g=\rho\left(\frac{\lambda}{\pi}\right)^{\frac{N+3}{2}} e^{-\lambda\left(\left(\xi_{x}-u\right)^{2}+\left(\xi_{y}-v\right)^{2}+\left(\xi_{z}-w\right)^{2}+\zeta^{2}\right)},
$$

where $\lambda=\rho / 2 p, \zeta^{2}=\zeta_{i} \zeta_{i}$. The internal degrees of freedom $N$ equals to 2 for diatomic gas.

In order to ensure that the BGK model (Eq. (2.3)) recovers the concerned N-S equations (Eq. (2.1)), from the Chapman-Enskog expansion analysis, the accelerations should be designed to satisfy the following equations

$$
-\int\left(\mathbf{a}_{\zeta} \cdot \nabla_{\xi} f\right) \boldsymbol{\varphi} d \Xi=[0,-\rho(\boldsymbol{\omega} \times \mathbf{v}), 0]^{T},
$$

where the notation $d \Xi=d \xi_{x} d \xi_{y} d \xi_{z} d \zeta_{1} d \zeta_{2} \cdots d \zeta_{N}$ has been used, and $\varphi$ is the vector of the collision invariants

$$
\boldsymbol{\varphi}=\left[1, \xi_{x}, \xi_{y}, \xi_{z}, \frac{1}{2}\left(\xi_{x}^{2}+\xi_{y}^{2}+\xi_{z}^{2}+\zeta^{2}\right)\right]^{T} .
$$

According to the theory of statistics, when $\xi_{x}, \xi_{y}, \xi_{z}, \zeta_{i} \rightarrow \pm \infty$, the distribution functions $f, g \rightarrow 0$ and both $f$ and $g$ are higher order infinitesimals. Therefore, two important integration relations are obtained, e.g., for the acceleration $a_{\xi, x}$

$$
\begin{aligned}
& \int a_{\xi, x} \frac{\partial f}{\partial \xi_{x}} \boldsymbol{\varphi} d \Xi=\int \frac{\partial a_{\tilde{\xi}, x} f \boldsymbol{\varphi}}{\partial \xi_{x}} d \Xi-\int \frac{\partial a_{\tilde{\xi}, x} \boldsymbol{\varphi}}{\partial \xi_{x}} f d \Xi=-\int \frac{\partial a_{\xi, x} \boldsymbol{\varphi}}{\partial \xi_{x}} f d \Xi, \\
& \int a_{\xi, x} \frac{\partial g}{\partial \xi_{x}} \boldsymbol{\varphi} d \Xi=\int \frac{\partial a_{\xi, x} g \boldsymbol{\varphi}}{\partial \xi_{x}} d \Xi-\int \frac{\partial a_{\xi, x} \boldsymbol{\varphi}}{\partial \xi_{x}} g d \Xi=-\int \frac{\partial a_{\xi, x} \boldsymbol{\varphi}}{\partial \xi_{x}} g d \Xi .
\end{aligned}
$$

The same holds for the other two accelerations $a_{\xi, y}$ and $a_{\xi, z}$. With these relations, Eq. (2.4) 
becomes (written in components)

$$
\begin{aligned}
& \int\left(\nabla_{\xi} \cdot \mathbf{a}_{\xi}\right) f d \Xi=0, \\
& \int\left[\xi_{x}\left(\nabla_{\xi} \cdot \mathbf{a}_{\xi}\right)+a_{\xi_{, x}}\right] f d \Xi=\rho\left(r_{y} w-r_{z} v\right), \\
& \int\left[\xi_{y}\left(\nabla_{\xi} \cdot \mathbf{a}_{\xi}\right)+a_{\xi_{, y}}\right] f d \Xi=\rho\left(r_{z} u-r_{x} w\right), \\
& \int\left[\xi_{z}\left(\nabla_{\xi} \cdot \mathbf{a}_{\xi}\right)+a_{\xi_{z}}\right] f d \Xi=\rho\left(r_{x} v-r_{y} u\right), \\
& \int\left[\frac{1}{2}\left(\xi_{x}^{2}+\xi_{y}^{2}+\xi_{z}^{2}\right)\left(\nabla_{\xi} \cdot \mathbf{a}_{\xi}\right)+\xi^{\prime} \cdot \mathbf{a}_{\xi}\right] f d \Xi=0,
\end{aligned}
$$

where the notation

$$
\nabla_{\xi} \cdot \mathbf{a}_{\xi}=\frac{\partial a_{\xi, x}}{\partial \xi_{x}}+\frac{\partial a_{\xi, y}}{\partial \xi_{y}}+\frac{\partial a_{\xi, z}}{\partial \xi_{z}}
$$

has been used. It is seen from Eq. (2.6) that there is not only one choice for determining $a_{\xi, x}, a_{\xi, y}$ and $a_{\xi, z}$. Here, we directly give an alternative and simple one

$$
\begin{aligned}
& a_{\xi, x}=\omega\left(r_{z} \xi_{y}-r_{y} \xi_{z}\right), \\
& a_{\xi, y}=\omega\left(r_{x} \xi_{z}-r_{z} \xi_{x}\right), \\
& a_{\xi, z}=\omega\left(r_{y} \xi_{x}-r_{x} \xi_{y}\right),
\end{aligned}
$$

where $r_{x}, r_{y}$ and $r_{z}$ are constants. It can be proven that with the above definition of $\mathbf{a}_{\tilde{\xi}}$, the connection between Eqs. (2.1) and (2.3) is built, where the compatibility condition between $f$ and $g$ needs to be used

$$
\int(f-g) \varphi d \Xi=0
$$

\subsection{Solution of the modified Boltzmann equation}

As the governing equation, the modified Boltzmann equation is solved at each cell interface for a second-order solution $f(\mathbf{x}, t, \xi, \zeta)$, from which the numerical fluxes can be evaluated. In the following, we take the $i$-direction face $\mathbf{x}_{i+1 / 2, j, k}=\left(x_{i+1 / 2, j, k}, y_{i+1 / 2, j, k}, z_{i+1 / 2, j, k}\right)$ as an example. Unless otherwise stated, the subscripts $j$ and $k$ will be both omitted. For convenience, the $x$-direction is assumed to be the normal direction to the local cell interface and the $y$-direction and $z$-direction are the other two tangential directions. The generalized solution of Eq. (2.3) at the cell interface $\mathbf{x}_{i+1 / 2}$ and at time $t$ can be written as

$$
f\left(\mathbf{x}_{i+1 / 2}, t, \xi, \zeta\right)=\frac{1}{\tau} \int_{0}^{t} e^{-\left(t-t^{\prime}\right) / \tau} g\left(\mathbf{x}\left(t^{\prime}\right), t^{\prime}, \xi, \zeta\right) d t^{\prime}+e^{-t / \tau} f_{0}(\mathbf{x}(0), \xi, \zeta),
$$

where $\boldsymbol{\xi}\left(t^{\prime}\right)=\boldsymbol{\xi}(t)-\left(t-t^{\prime}\right) \mathbf{a}_{\xi}$ and $\mathbf{x}\left(t^{\prime}\right)=\mathbf{x}_{i+1 / 2}-\xi^{\prime}\left(t-t^{\prime}\right)-\frac{1}{2} \mathbf{a}_{\xi}\left(t-t^{\prime}\right)^{2}$ are the velocity and trajectory of a particle motion with $t^{\prime} \in[0, t]$, due to the non-inertial forces. Because the 
term $-\frac{1}{2} \mathbf{a}_{\tilde{\zeta}}\left(t-t^{\prime}\right)^{2}$ has only a higher-order effect, it can be ignored. The integral solution $f$ describes a dynamic process of gas evolution, starting with an initial gas distribution function $f_{0}$ and approaching the equilibrium state $g$. In the next, we will demonstrate how to evaluate $f_{0}$ and $g$, respectively. For simplicity, the location of the cell interface is assumed to be $\mathbf{x}_{i+1 / 2}=(0,0,0)$.

\subsubsection{The initial state $f_{0}$}

In order to develop a multidimensional BGK scheme, $f_{0}$ can be constructed as

$$
\begin{aligned}
f_{0} & =g-\left(\boldsymbol{\xi}^{\prime} \cdot \nabla_{g}\right) t-\left(\mathbf{a}_{\xi} \cdot \nabla_{\xi} g\right) t-\tau\left(\frac{\partial g}{\partial t}+\nabla g \cdot \xi^{\prime}+\mathbf{a}_{\xi} \cdot \nabla_{\xi} g\right) \\
& = \begin{cases}g^{l}\left[1-\left(\mathbf{a}^{l} \cdot \boldsymbol{\xi}^{\prime}\right) t-\tau\left(A^{l}+\mathbf{a}^{l} \cdot \boldsymbol{\xi}^{\prime}\right)\right]-(t+\tau)\left(\mathbf{a}_{\xi} \cdot \nabla_{\xi} g^{l}\right), & x<0 \Leftrightarrow \xi^{\prime}{ }_{x}>0, \\
g^{r}\left[1-\left(\mathbf{a}^{r} \cdot \xi^{\prime}\right) t-\tau\left(A^{r}+\mathbf{a}^{r} \cdot \xi^{\prime}\right)\right]-(t+\tau)\left(\mathbf{a}_{\xi} \cdot \nabla_{\xi} g^{r}\right), & x \geq 0 \Leftrightarrow \xi^{\prime}{ }_{x} \leq 0,\end{cases}
\end{aligned}
$$

where $g^{l}$ and $g^{r}$ are local Maxwellians at the left and right sides of the cell interface, $\mathbf{a}^{l(r)}=\left[a^{l(r)}, b^{l(r)}, c^{l(r)}\right]$ and $A^{l(r)}$ are related to derivatives of $g^{l(r)}$ in space and time. All the terms involving $\tau$ denote the non-equilibrium parts in $f_{0}$, including the additional acceleration term. As shown previously, the equilibrium states $g^{l(r)}$ are obtained from the reconstructed macroscopic flow variables at both sides of the cell interface, i.e., $\mathbf{W}^{l}$ and $\mathbf{W}^{r}$. The slopes $a^{l(r)}, b^{l(r)}$ and $c^{l(r)}$ are evaluated as follows. For example, when taking the derivatives of $g^{l}$ in the normal direction, i.e., when expanding $\frac{\partial g^{l}}{\partial x}=a^{l} g^{l}$, it is found that $a^{l}$ can be expressed as a linear combination of the collision invariants $\varphi_{\alpha},(\alpha=1 \sim 5)$. The same holds for the other slopes and therefore

$$
\begin{aligned}
& a^{l(r)}=a_{\alpha}^{l(r)} \varphi_{\alpha}, \\
& b^{l(r)}=b_{\alpha}^{l(r)} \varphi_{\alpha}, \\
& c^{l(r)}=c_{\alpha}^{l(r)} \varphi_{\alpha} .
\end{aligned}
$$

All the unknown coefficients $a_{\alpha}^{l(r)}, b_{\alpha}^{l(r)}$ and $c_{\alpha}^{l(r)}$ are local constants related to macroscopic variables and their gradients. We can directly obtain these coefficients by using the chain rule for the Maxwellian distribution function (see Appendix), however, a more efficient way is to evaluate them implicitly. According to the conservation forms of moments $\mathbf{W}=\int g \boldsymbol{\varphi} d \Xi$ and noticing that $d \Xi$ is independent of $x, y$ and $z$, we get

$$
\begin{aligned}
\int g^{l(r)} a_{\alpha}^{l(r)} \varphi_{\alpha} \varphi d \Xi & =\left(\frac{\partial \mathbf{W}}{\partial x}\right)^{l(r)}, \\
\int g^{l(r)} b_{\alpha}^{l(r)} \varphi_{\alpha} \varphi d \Xi & =\left(\frac{\partial \mathbf{W}}{\partial y}\right)^{l(r)}, \\
\int g^{l(r)} c_{\alpha}^{l(r)} \varphi_{\alpha} \varphi d \Xi & =\left(\frac{\partial \mathbf{W}}{\partial z}\right)^{l(r)},
\end{aligned}
$$


where the terms on the right side represent the gradients of the (reconstructed) conservative variables around the cell interface. By solving the above linear systems, all the slopes are obtained, which are consistent with those shown in Appendix. The calculations for $\mathbf{W}^{l(r)}$ and the derivatives will be presented in Section 2.3. The parameters $A^{l(r)}$ can be similarly expressed as

$$
A^{l(r)}=A_{\alpha}^{l(r)} \varphi_{\alpha} .
$$

Then considering the fact that the non-equilibrium parts in $f_{0}$ have no direct contributions to conservative variables, i.e.,

$$
\int \tau\left[g\left(A^{l(r)}+\mathbf{a}^{l(r)} \cdot \xi^{\prime}\right)+\mathbf{a}_{\xi} \cdot \nabla_{\xi} g^{l(r)}\right] \varphi d \Xi=0,
$$

we further have

$$
\int g A_{\alpha}^{l(r)} \varphi_{\alpha} \boldsymbol{\varphi} d \Xi=-\int g\left(\mathbf{a}^{l(r)} \cdot \boldsymbol{\xi}^{\prime}\right) \boldsymbol{\varphi} d \Xi-\int\left(\mathbf{a}_{\xi} \cdot \nabla_{\xi} g^{l(r)}\right) \boldsymbol{\varphi} d \Xi .
$$

The first term on the right side of the above equation can be easily obtained since all the slopes are known. The second term can be calculated from Eqs. (2.5) and (2.7), i.e.,

$$
\int\left(\mathbf{a}_{\zeta} \cdot \nabla_{\xi} g^{l(r)}\right) \boldsymbol{\varphi} d \Xi=\left[0, \rho^{l(r)}\left(\boldsymbol{\omega} \times \mathbf{v}^{l(r)}\right), 0\right]^{T} .
$$

By solving two linear systems (Eq. (2.15)), $A^{l}$ and $A^{r}$ are both determined.

\subsubsection{The equilibrium state $g$}

Different from most existing work, the equilibrium state $g$ around the cell interface and time $t=0$ is constructed as a more general formulation with consideration of the possible discontinuities of tangential gradients at the cell interface

$$
g=g_{0}\left\{1+\bar{A} t^{\prime}-\left[H\left(\xi^{\prime}{ }_{x}\right)\left(\overline{\mathbf{a}}^{l} \cdot \xi^{\prime}\right)+\left(1-H\left(\xi^{\prime}{ }_{x}\right)\right)\left(\overline{\mathbf{a}}^{r} \cdot \xi^{\prime}\right)\right]\left(t-t^{\prime}\right)\right\}-\left(\mathbf{a}_{\xi} \cdot \nabla_{\xi} g_{0}\right)\left(t-t^{\prime}\right),
$$

where $H\left(\tilde{\zeta}_{x}^{\prime}\right)$ is the Heaviside function. The parameters $\overline{\mathbf{a}}^{l(r)}=\left[\bar{a}^{l(r)}, \bar{b}^{l(r)}, \bar{c}^{l(r)}\right]$ and $\bar{A}$ are related to derivatives of $g_{0}$ in space and time. The equilibrium state $g_{0}$ (at time $t=0$ ) is also a Maxwellian

$$
g_{0}=\bar{\rho}\left(\frac{\bar{\lambda}}{\pi}\right)^{\frac{N+3}{2}} e^{-\bar{\lambda}\left(\left(\tilde{\zeta}_{x}-\bar{u}\right)^{2}+\left(\tilde{\zeta}_{y}-\bar{v}\right)^{2}+\left(\tilde{\zeta}_{z}-\bar{w}\right)^{2}+\zeta^{2}\right)},
$$

where the unknown macroscopic variables $\bar{\rho}, \bar{\lambda}, \bar{u}, \bar{v}$ and $\bar{w}$ in $g_{0}$ can be obtained by taking the limits of $\mathbf{x} \rightarrow(0,0,0)$ and $t \rightarrow 0$ in Eqs. (2.9) and (2.10) and using Eq. (2.14), which gives

$$
\overline{\mathbf{W}}=\int g_{0} \boldsymbol{\varphi} d \Xi=\int\left[H\left(\tilde{\xi}_{x}^{\prime}\right) g^{l}+\left(1-H\left(\xi^{\prime}{ }_{x}\right)\right) g^{r}\right] \varphi d \Xi,
$$


where $\overline{\mathbf{W}}$ is defined as the "average" of conservative variables at the cell interface. In the above equation, the integrations for $\xi_{x}=\xi_{x}^{\prime}{ }_{x}+u_{e}$ are done in two non-symmetric velocity spaces. With such bounded integration limits, the moments of the Maxwellian distribution function for $\xi_{x}^{n},(n=0,1,2, \cdots)$ can be simply obtained by using the error function and the complementary error function. In order to determine $\bar{a}^{l(r)}, \bar{b}^{l(r)}$ and $\bar{c}^{l(r)}$, expanding them as

$$
\begin{aligned}
& \bar{a}^{l(r)}=\bar{a}_{\alpha}^{l(r)} \varphi_{\alpha}, \\
& \bar{b}^{l(r)}=\bar{b}_{\alpha}^{l(r)} \varphi_{\alpha}, \\
& \bar{c}^{l(r)}=\bar{c}_{\alpha}^{l(r)} \varphi_{\alpha} .
\end{aligned}
$$

The coefficients $\bar{a}_{\alpha}^{l(r)}, \bar{b}_{\alpha}^{l(r)}$ and $\bar{c}_{\alpha}^{l(r)}$ are obtained from the following equations

$$
\begin{aligned}
\int g_{0} \bar{a}_{\alpha}^{l(r)} \varphi_{\alpha} \varphi d \Xi & =\left(\frac{\partial \overline{\mathbf{W}}}{\partial x}\right)^{l(r)}, \\
\int g_{0} \bar{b}_{\alpha}^{l(r)} \varphi_{\alpha} \boldsymbol{\varphi} d \Xi & =\left(\frac{\partial \overline{\mathbf{W}}}{\partial y}\right)^{l(r)}, \\
\int g_{0} \bar{c}_{\alpha}^{l(r)} \varphi_{\alpha} \varphi d \Xi & =\left(\frac{\partial \overline{\mathbf{W}}}{\partial z}\right)^{l(r)},
\end{aligned}
$$

where the terms on the right side represent the gradients of $\overline{\mathbf{W}}$ around the cell interface. The calculations for them will be together discussed in Section 2.3.

\subsubsection{The expressions of the solution $f$ and time-average fluxes}

With the above definitions of $f_{0}$ and $g$, according to Eq. (2.9), the solution $f$ of the constructed BGK model can be written as

$$
\begin{aligned}
& f\left(\mathbf{x}_{i+1 / 2}, t, \xi_{,}, \zeta\right) \\
& =\gamma_{1} g_{0}+\gamma_{2} \bar{A} g_{0}+\gamma_{3}\left[H\left(\xi^{\prime}{ }_{x}\right) g_{0}\left(\overline{\mathbf{a}}^{l} \cdot \xi^{\prime}\right)+\left(1-H\left(\xi^{\prime}{ }_{x}\right)\right) g_{0}\left(\overline{\mathbf{a}}^{r} \cdot \xi^{\prime}\right)+\mathbf{a}_{\xi} \cdot \nabla_{\xi} g_{0}\right] \\
& \quad+\gamma_{4}\left[H\left(\xi^{\prime}{ }_{x}\right) g^{l}\left(1-\tau A^{l}\right)+\left(1-H\left(\xi^{\prime}{ }_{x}\right) g^{r}\left(1-\tau A^{r}\right)\right]\right. \\
& \quad+\gamma_{5}\left[H\left(\xi^{\prime}{ }_{x}\right)\left(\left(\mathbf{a}^{l} \cdot \xi^{\prime}\right) g^{l}+\mathbf{a}_{\xi} \cdot \nabla_{\xi} g^{l}\right)+\left(1-H\left(\xi^{\prime}{ }_{x}\right)\right)\left(\left(\mathbf{a}^{r} \cdot \xi^{\prime}\right) g^{r}+\mathbf{a}_{\xi} \cdot \nabla_{\xi} g^{r}\right)\right]
\end{aligned}
$$

with the coefficients $\gamma_{1}, \gamma_{2}, \cdots, \gamma_{5}$ defined as

$$
\begin{array}{ll}
\gamma_{1}=1-e^{-t / \tau}, & \gamma_{2}=t-\tau\left(1-e^{-t / \tau}\right), \\
\gamma_{4}=e^{-t / \tau}, & \gamma_{5}=-(t+\tau) e^{-t / \tau} .
\end{array}
$$

Due to the presence of $\nabla_{\xi} g$, it is difficult to construct the acceleration term $\left(\mathbf{a}_{\xi} \cdot \nabla_{\xi g}\right)$, let alone explicitly gain the values of $f_{0}, g$ and $f$. Fortunately, the finite volume BGK method 
actually focuses on the evaluation of the numerical fluxes at the cell interface by reconstructing the gas distribution function $f$, thus we only need to calculate the moments of the acceleration term (or the terms including it). For example, by multiplying $\left(\mathbf{a}_{\xi} \cdot \nabla_{\xi g}\right)$ by $\xi^{\prime}{ }_{x}$ and $\varphi_{\alpha},(\alpha=1 \sim 5)$ and then integrating it with respect to $d \Xi$, according to Eqs. (2.5) and (2.7), the acceleration term-related fluxes become

$$
\int \xi^{\prime}{ }_{x} \varphi_{\alpha}\left(\mathbf{a}_{\xi} \cdot \nabla_{\xi} g\right) d \Xi=-\int g\left[\mathbf{a}_{\xi} \cdot \nabla_{\xi}\left(\xi^{\prime}{ }_{x} \varphi_{\alpha}\right)\right] d \Xi
$$

Now the only unknown in Eq. (2.21) is $\bar{A}$. It is noted that both the expressions of $f$ and $g$ contain $\bar{A}$, therefore by using $\bar{A}=\bar{A}_{\alpha} \varphi_{\alpha}$ and integrating the compatibility condition (Eq. (2.8)) over a whole time step $\Delta t$, i.e.,

$$
\int_{0}^{\Delta t} \int(f-g)^{\prime} d \Xi=0
$$

we can get $\bar{A}$ from the above linear equations. As a common practice, for simulation of turbulent flows [21,22], the collision time $\tau$ in $f$ is computed by

$$
\tau=\frac{\mu_{L}+\mu_{T}}{p}+c \Delta t \frac{\left|p_{l}-p_{r}\right|}{\left|p_{l}+p_{r}\right|}
$$

where $\mu_{L}$ and $\mu_{T}$ denote the laminar viscosity and eddy viscosity at the cell interface, respectively. The eddy viscosity $\mu_{T}$ is obtained by employing a turbulence model, such as the Spalart-Allmaras one-equation model used here [23]. The presence of the additional dissipation term is numerically necessary to capture a stable and robust shock structure, where $\Delta t$ is the computational time step satisfying the CFL condition and $c$ usually takes a value ranging from 1 to 5 .

It is seen from Eq. (2.21) that the solution $f$ is time-dependent, and a uniform $\Delta t$ throughout the flow domain is necessary, which takes the minimum value among all the local time steps. This, however, may significantly decrease the computational efficiency for steady-state flow computations. Considering that the BGK scheme focuses on evaluation of fluxes at the cell interface, the local time step $\Delta t$ is still used to accelerate convergence, and the time-average fluxes $\mathbf{F}$ are introduced

$$
\mathbf{F}=\frac{1}{\Delta t} \iint \xi^{\prime}{ }_{x} f\left(\mathbf{x}, t,{ }_{,}, \zeta\right)^{\prime} d \Xi d t
$$

An efficient BGK-based implicit scheme by using the Jacobian-free Newton-Krylov method [24] is employed for update of flow variables. The Chapman-Enskog expansion shows that the developed BGK model only recovers the N-S equations with a fixed Prandtl number of 1 . In order to simulate flows with arbitrary Prandtl number, a simple but effective way is adopted by approximately modifying the heat fluxes [25]. 


\subsection{Calculations of $\mathbf{a}^{l(r)}$ and $\overline{\mathbf{a}}^{l(r)}$}

As analyzed in [13], the viscous part of fluxes are contributed by a nonlinear average of spatial derivatives of the initial state $f_{0}$ and the equilibrium state $g$, i.e., the terms related to $\mathbf{a}^{l(r)}=\left[a^{l(r)}, b^{l(r)}, c^{l(r)}\right]^{T}$ and $\overline{\mathbf{a}}^{l(r)}=\left[\bar{a}^{l(r)}, \bar{b}^{l(r)}, \bar{c}^{l(r)}\right]^{T}$ in Eq. (2.21). This section addresses how to calculate these slopes in a more robust and accurate way since it is found that the computed results for viscous flows could be greatly dependent on them. Before that, one should be noted that the coordinates $(x, y, z)$ used in this section represent the global coordinate system, instead of the local system established previously. And it is easy to convert the flow quantities between these two systems.

From Eqs. (2.12) and (2.20), the key point is to obtain the gradients of flow variables $\mathbf{W}$ and $\overline{\mathbf{W}}$. In most BGK schemes, a simple finite difference is employed, e.g., for $(\partial \mathbf{W} / \partial x)^{l(r)}$

$$
\begin{aligned}
& \left(\frac{\partial \mathbf{W}}{\partial x}\right)^{l}=\frac{\mathbf{W}_{i+1 / 2}^{l}-\mathbf{W}_{i}}{x_{i+1 / 2}-x_{i}}=\frac{\mathbf{W}_{i+1 / 2}^{l}-\mathbf{W}_{i-1 / 2}^{r}}{x_{i+1 / 2}-x_{i-1 / 2}} \\
& \left(\frac{\partial \mathbf{W}}{\partial x}\right)^{r}=\frac{\mathbf{W}_{i+1}-\mathbf{W}_{i+1 / 2}^{r}}{x_{i+1}-x_{i+1 / 2}}=\frac{\mathbf{W}_{i+3 / 2}^{l}-\mathbf{W}_{i+1 / 2}^{r}}{x_{i+3 / 2}-x_{i+1 / 2}}
\end{aligned}
$$

and for $(\partial \overline{\mathbf{W}} / \partial x)^{l(r)}$,

$$
\begin{aligned}
& \left(\frac{\partial \overline{\mathbf{W}}}{\partial x}\right)^{l}=\frac{\overline{\mathbf{W}}_{i+1 / 2}-\mathbf{W}_{i}}{x_{i+1 / 2}-x_{i}}, \\
& \left(\frac{\partial \overline{\mathbf{W}}}{\partial x}\right)^{r}=\frac{\mathbf{W}_{i+1}-\overline{\mathbf{W}}_{i+1 / 2}}{x_{i+1}-x_{i+1 / 2}},
\end{aligned}
$$

where $\mathbf{W}_{i}$ and $\mathbf{W}_{i+1}$ represent the cell-averaged conservative variables at the left and right cells. However, there are two disadvantages when using Eqs. (2.26) and (2.27). The first one is that the above relations may give inadequate values for non-Cartesian mesh so that they cannot be directly used. The detailed analysis have been provided in [13], where it is also pointed out that the incorrect gradients can have disastrous effects. Although we can remove this problem through the use of a coordinate transformation or a modified approach [13], it is still found that the way to calculate the gradients by a difference approximation sometimes leads to unphysical results and even numerical instabilities, which is thought as the second disadvantage. The reason is that the computed values may be strongly sensitive to the mesh quality, or more specifically, the distance between cell centers and the distance between the cell center and the cell interface. This phenomenon can be especially severe in practical rotating fluid applications such as in turbomachinery. In order to improve the robustness and accuracy for calculating the derivatives of $\mathbf{W}$ and $\overline{\mathbf{W}}$ and the subsequent $\mathbf{a}^{l(r)}$ and $\overline{\mathbf{a}}^{l(r)}$, an improved approach is adopted.

Based on the MUSCL approach, a second-order upwind-biased scheme is first used to obtain the left macroscopic state $\mathbf{W}^{l}$ and the right macroscopic state $\mathbf{W}^{r}$ at the cell interface. To prevent the generation of oscillation and spurious solutions in discontinuous 
regions, the Van Albada's limiter [26] is together employed. Therefore, for each $W^{l}(\alpha)$ and $W^{r}(\alpha),(\alpha=1 \sim 5)$, we have (for short, $(\alpha)$ is omitted)

$$
\begin{aligned}
& W^{l}=W_{i}+\frac{1}{2} \frac{\Delta^{+} W_{i} \Delta^{-} W_{i}\left(\Delta^{+} W_{i}+\Delta^{-} W_{i}\right)+\varepsilon}{\left(\Delta^{+} W_{i}\right)^{2}+\left(\Delta^{-} W_{i}\right)^{2}+\varepsilon} \\
& W^{r}=W_{i+1}-\frac{1}{2} \frac{\Delta^{+} W_{i+1} \Delta^{-} W_{i+1}\left(\Delta^{+} W_{i+1}+\Delta^{-} W_{i+1}\right)+\varepsilon}{\left(\Delta^{+} W_{i+1}\right)^{2}+\left(\Delta^{-} W_{i+1}\right)^{2}+\varepsilon}
\end{aligned}
$$

where $\Delta^{+} W_{i}=W_{i+1}-W_{i}, \Delta^{-} W_{i}=W_{i}-W_{i-1}, \varepsilon$ is a small quantity. The above equations can be similarly applied in the $j-$ and $k$ - directions. Then the derivatives of $\mathbf{W}$ are calculated by applying the Gauss-Green theorem to the left and right cells, e.g., for $(\partial \mathbf{W} / \partial x)^{l(r)}$,

$$
\begin{aligned}
\left(\frac{\partial \mathbf{W}}{\partial x}\right)^{l}= & \frac{1}{\Omega_{i, j, k}}\left[\left(\mathbf{W}^{l} S_{x}\right)_{i+1 / 2, j, k}+\left(\mathbf{W}^{l} S_{x}\right)_{i, j+1 / 2, k}+\left(\mathbf{W}^{l} S_{x}\right)_{i, j, k+1 / 2}\right. \\
& \left.-\left(\mathbf{W}^{r} S_{x}\right)_{i-1 / 2, j, k}-\left(\mathbf{W}^{r} S_{x}\right)_{i, j-1 / 2, k}-\left(\mathbf{W}^{r} S_{x}\right)_{i, j, k-1 / 2}\right] \\
\left(\frac{\partial \mathbf{W}}{\partial x}\right)^{r}= & \frac{1}{\Omega_{i+1, j, k}}\left[\left(\mathbf{W}^{l} S_{x}\right)_{i+3 / 2, j, k}+\left(\mathbf{W}^{l} S_{x}\right)_{i+1, j+1 / 2, k}+\left(\mathbf{W}^{l} S_{x}\right)_{i+1, j, k+1 / 2}\right. \\
& \left.-\left(\mathbf{W}^{r} S_{x}\right)_{i+1 / 2, j, k}-\left(\mathbf{W}^{r} S_{x}\right)_{i+1, j-1 / 2, k}-\left(\mathbf{W}^{r} S_{x}\right)_{i+1, j, k-1 / 2}\right]
\end{aligned}
$$

where $\Omega$ is the volume, and $S_{x}$ is the $x$-component of the face vector. Likewise, because the "average" conservative variables $\overline{\mathbf{W}}$ have been obtained at each cell interface, the derivatives of $\overline{\mathbf{W}}$ can be calculated as follow

$$
\begin{aligned}
\left(\frac{\partial \overline{\mathbf{W}}}{\partial x}\right)^{l}= & \frac{1}{\Omega_{i, j, k}}\left[\left(\overline{\mathbf{W}} S_{x}\right)_{i+1 / 2, j, k}+\left(\overline{\mathbf{W}} S_{x}\right)_{i, j+1 / 2, k}+\left(\overline{\mathbf{W}} S_{x}\right)_{i, j, k+1 / 2}\right. \\
& \left.-\left(\overline{\mathbf{W}} S_{x}\right)_{i-1 / 2, j, k}-\left(\overline{\mathbf{W}} S_{x}\right)_{i, j-1 / 2, k}-\left(\overline{\mathbf{W}} S_{x}\right)_{i, j, k-1 / 2}\right] \\
\left(\frac{\partial \overline{\mathbf{W}}}{\partial x}\right)^{r}= & \frac{1}{\Omega_{i+1, j, k}}\left[\left(\overline{\mathbf{W}} S_{x}\right)_{i+3 / 2, j, k}+\left(\overline{\mathbf{W}} S_{x}\right)_{i+1, j+1 / 2, k}+\left(\overline{\mathbf{W}} S_{x}\right)_{i+1, j, k+1 / 2}\right. \\
& \left.-\left(\overline{\mathbf{W}} S_{x}\right)_{i+1 / 2, j, k}-\left(\overline{\mathbf{W}} S_{x}\right)_{i+1, j-1 / 2, k}-\left(\overline{\mathbf{W}} S_{x}\right)_{i+1, j, k-1 / 2}\right] .
\end{aligned}
$$

When $S_{x}$ in the above two equations is replaced with $S_{y}$ or $S_{z}$, the derivatives in the $y-$ and $z$ - directions can also be evaluated. In order to make the illustrations more clear, Fig. 1 shows the schematics for evaluation of gradients of $\mathbf{W}$ and $\overline{\mathbf{W}}$. Although for clarity only the two-dimensional case is presented, one can easily extend it to the three-dimensional case, which exactly leads to Eqs. (2.28) and (2.29). Numerically, it is shown that in the case of Cartesian mesh, the current approach has the same form as Eqs. (2.26) and (2.27). However, it can better deal with non-Cartesian mesh even of poor quality as the sensitivity of the calculated results to mesh quality is reduced. And due to this, the robustness of the gas-kinetic BGK scheme can be improved. Further, it is 

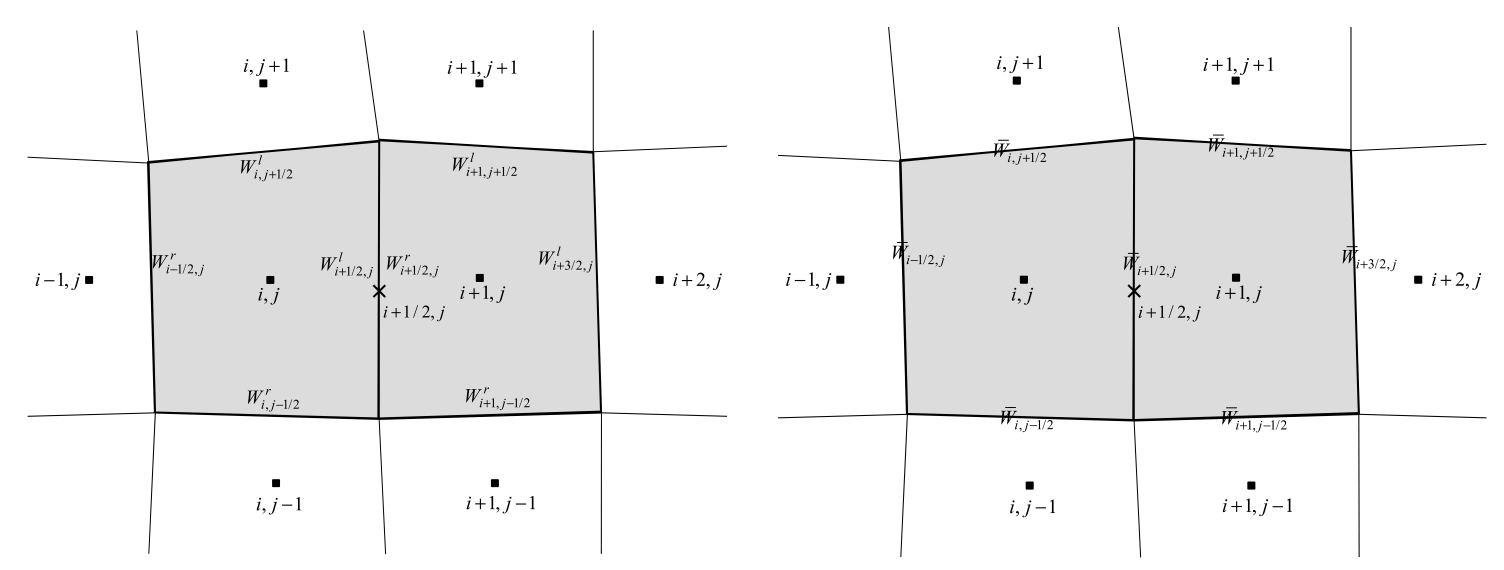

Figure 1: Schematics for evaluation of gradients of (a) $\mathbf{W}$ and (b) $\overline{\mathbf{W}}$ for the two-dimensional case as an example.

seen from Eq. (2.21) that for flows of moderate Reynolds numbers $(\tau \ll t)$, the spatial derivatives of $g$ play a dominant role in contributing to the viscous part of fluxes, thus Eq. (2.29) should accurately reflect the viscous effects. We know that in the conventional N-S solvers, a usual way for evaluation of viscous fluxes is as follows. First, the cellcentered flow variables are averaged at cell interfaces, then the gradients for each cell are obtained by applying the Gauss-Green theorem, finally they are simply averaged at cell interfaces. This way actually implies an assumption that the gradients of any flow variables are continuous at the cell interface. In the current approach, the first two steps are also needed as indicated in Eqs. (2.18) and (2.29), however, the gradients of $g$ in normal and tangential directions are allowed to be discontinuous. Physically, it describes the formation of a continuous equilibrium state $g$ at the interface through a collision process, which reflects the microcosmic mechanism of the real viscosity.

\section{Results and discussions}

For validation, several numerical examples are presented, including a rotating cavity with radial outflow, a rotating cavity with axial throughflow, a low-speed centrifugal compressor and a transonic axial-flow fan rotor. In all computations, a non-inertial frame of reference tied to the rotating parts is used. The laminar Prandtl number is taken as 0.72 and the turbulent Prandtl number is taken as 0.9.

Case 1: Rotating cavity with radial outflow.

A cavity formed between two co-rotating disks with an outer shroud is a common feature in rotating machinery, such as in compressor and turbine rotor assemblies. The study of flows in rotating cavities can be of great importance to the predictions of disk stress and component life. In this case, the incompressible laminar flow inside a rotating 

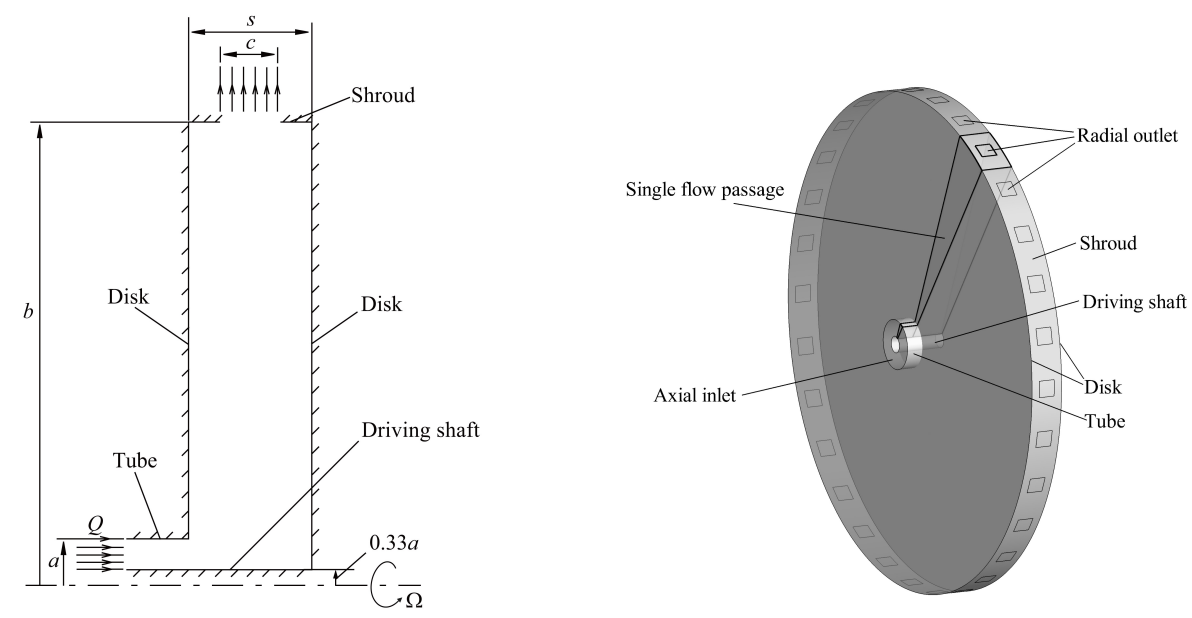

Figure 2: Schematic diagrams of a rotating cavity with radial outflow.

cavity with radial outflow is considered. An idealized model of such flow is shown in Fig. 2, which has been experimentally and theoretically studied by Owen et al. [27]. This cavity corresponds to rig (2.3) used for the outflow experiments, which has axial width $s=50.7 \mathrm{~mm}$, inner radius $a=38.1 \mathrm{~mm}$ and outer radius $b=381 \mathrm{~mm}$. There are a total of 30 square holes with a side of length $c=25.4 \mathrm{~mm}$ uniformly distributed in the whole shroud, through which the air is extracted from the cavity. A central driving shaft with diameter $0.66 a$ is fitted through the tube. For the axial inlet case, the inlet velocity is determined from the volume flow rate of the fluid entering the cavity $Q=C_{w} v b$, where $C_{w}$ is the volume flow-rate coefficient and $v$ is the kinematic viscosity. The whole assembly including two disks, the tube, the shroud and the driving shaft is rotating at an angular speed $\Omega$, which is determined by $\Omega=\operatorname{Re}_{\phi} v / b^{2}$ ( $R e_{\phi}$ is the rotational Reynolds number).

As shown in Fig. 2(b), the computational domain can be reduce to a single flow passage owing to the flow periodicity in circumferential direction. At the inlet, the complete absolute velocity vector and the temperature are specified while the pressure is extrapolated from the interior. At the outlet, the pressure is specified while the other variables are obtained from the interior. The periodic conditions are applied to circumferential boundaries. The no-slip isothermal boundary conditions are applied to the rotating walls, and the whole flow field can be approximately thought to be isothermal. The computational mesh is composed of two H-type blocks with sizes of $41 \times 61 \times 61$ and $81 \times 61 \times 161$, respectively.

Fig. 3 first shows the in-plane streamlines at mid-circumferential plane for (a) $R e_{\phi}=$ $5 \times 10^{4}, C_{w}=253$ and (b) $C_{w}=487$. The predicted streamline pattern is consistent with the flow visualization, numerically supporting the theoretical source-sink flow model. The flow can be divided into four types of regions. The viscous regions between the interior core and the disk faces are referred to as Ekman layers, where the steady boundary layers 


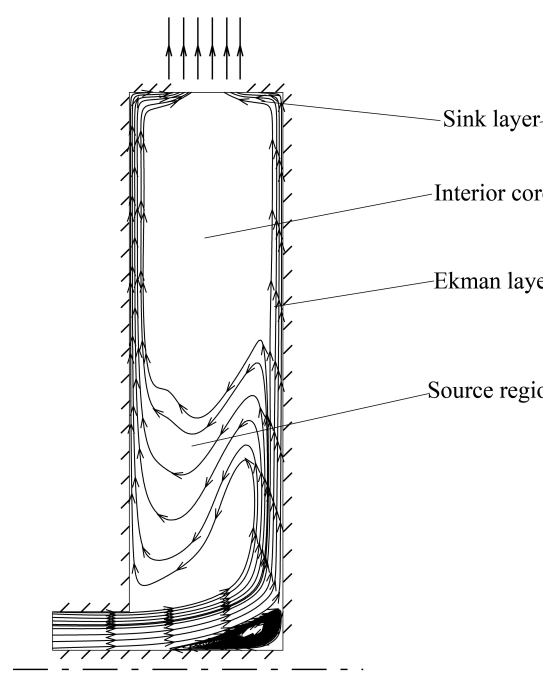

(a) $C_{w}=253$

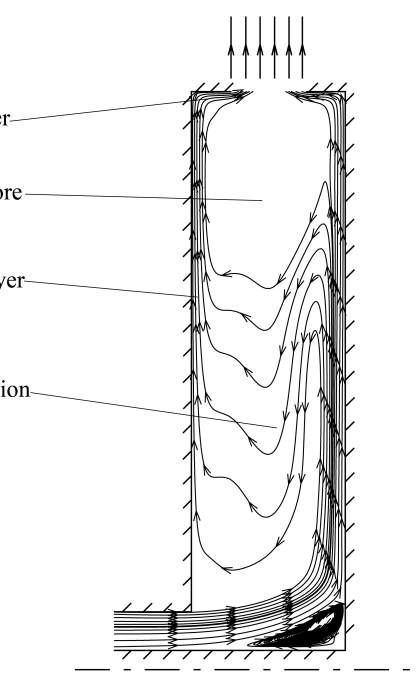

(b) $C_{w}=487$

Figure 3: The in-plane streamlines at mid-circumferential plane for $R e_{\phi}=5 \times 10^{4}$ and two different values of $C_{w}$.

become "non-entraining". The interior core appears between the two disk face boundary layer flows, where viscous forces are negligible. The source region defines the zone where the fluid is distributed from the source to the Ekman layers. On the contrary, the sink layer redistributes the flow from the Ekman layers to the exit. Comparing the results for $C_{w}=253$ and $C_{w}=487$, similar flow structures are found. However, with the increase of the volume flow rate, the source region becomes larger while the interior core becomes smaller, which has also been experimentally observed. Fig. 4 gives the corresponding inplane velocity contours. It is found that in the interior core, the radial and axial velocities are very small and thus the fluid almost purely rotates at a certain angular speed. For the current case of radial outflow, this speed is less than that of the disk, which can be seen below.

The computed radial variations of $v_{\phi} / \Omega r\left(v_{\phi}\right.$ is the absolute tangential velocity) at mid-axial plane for the above two conditions are presented in Fig. 5, where the predicted values are less than 1 at all radial locations. Also presented in this figure are the results from the experiment and from the nonlinear theory [27]. It is found that in the interior core $\left(0.98 \geq r / b \geq 0.6\right.$ for $C_{w}=253$ and $0.98 \geq r / b \geq 0.75$ for $\left.C_{w}=487\right)$, the numerical, theoretical and experimental results agree well with each other, and in the source region $\left(r / b<0.75\right.$ for $\left.C_{w}=487\right)$, the computed velocity distribution is still consistent with the experimental data. The discrepancies between the computed and theoretical results at near-shroud locations are possibly due to the effect of the shroud hole on the local flow field. In fact, without consideration of the "hole effect", the flow can be thought to be 


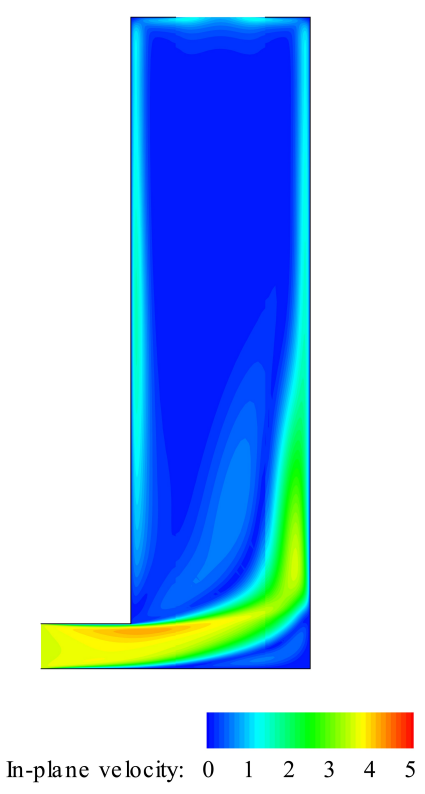

(a) $C_{w}=253$

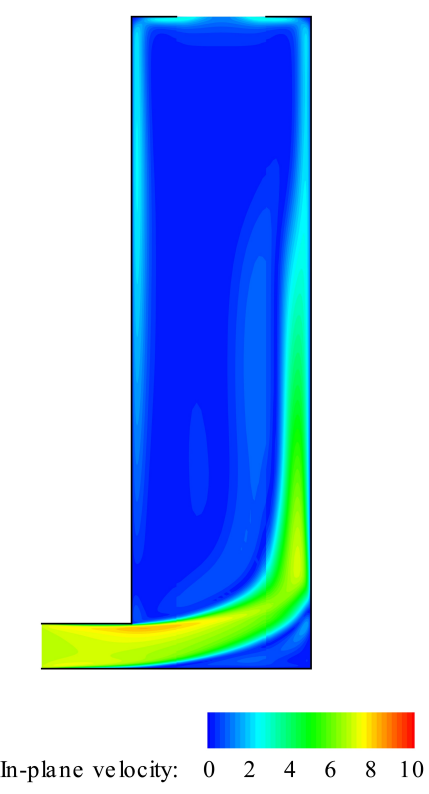

(b) $C_{w}=487$

Figure 4: The in-plane velocity contours at mid-circumferential plane for $R e_{\phi}=5 \times 10^{4}$ and two different values of $C_{w}$.

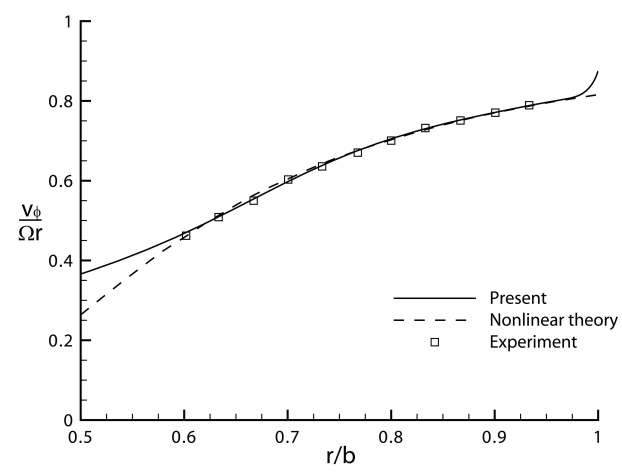

(a) $C_{w}=253$

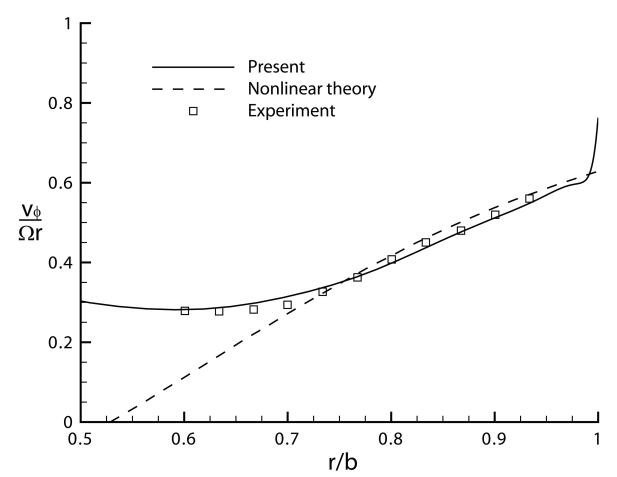

(b) $C_{w}=487$

Figure 5: The radial variations of $v_{\phi} / \Omega r$ at mid-axial plane for $R e_{\phi}=5 \times 10^{4}$ and two different values of $C_{w}$.

axisymmetric as assumed in the nonlinear theory.

Case 2: Rotating cavity with axial throughflow.

This case illustrates a rotating cavity with axial throughflow, which is often used to model part of the stack formed by multiple disks in an axial flow compressor. Fig. 6 

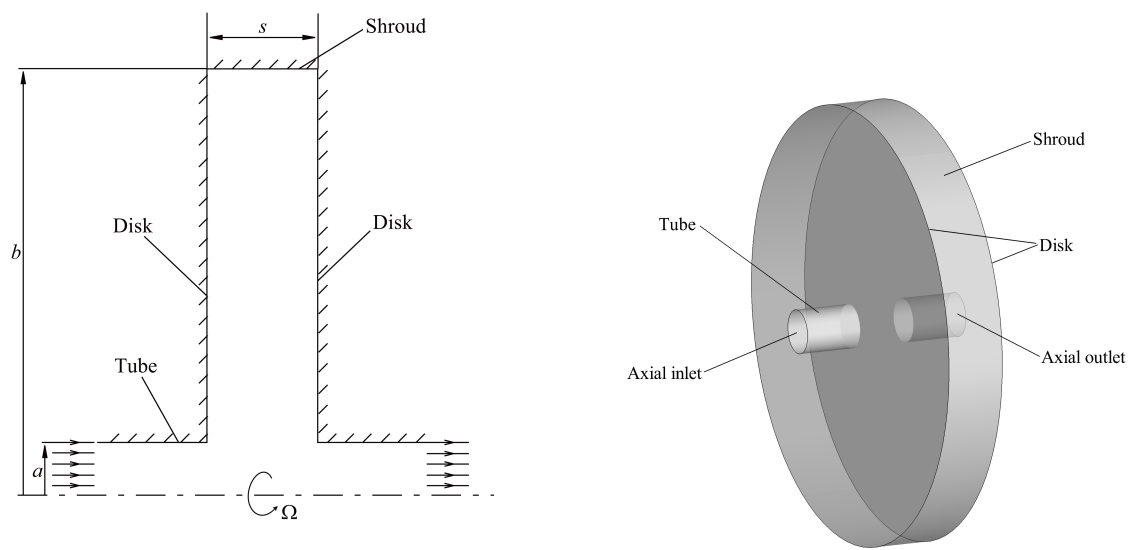

Figure 6: Schematic diagrams of a rotating cavity with axial throughflow.

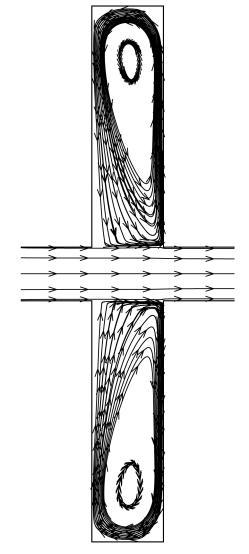

(a) $\operatorname{Ro} \rightarrow \infty$

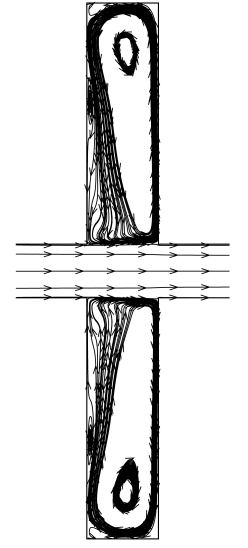

(b) $R o=160$

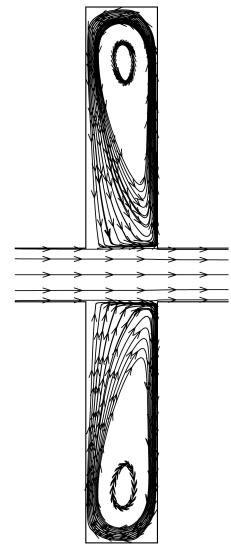

(c) $R o=80$

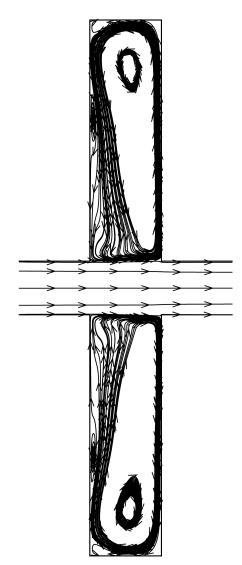

(d) $R o=40$

Figure 7: Schematic diagrams of a rotating cavity with axial throughflow.

shows a simplified model with the inner radius of two disks $a=19 \mathrm{~mm}$, the outer radius $b=190 \mathrm{~mm}$ and the axial gap $s=50.73 \mathrm{~mm}$, corresponding to the cavity with a gap ratio of $G=s / b=0.267$ conducted in rig (b) [28]. In numerical computations, the inlet and outlet boundary conditions are imposed the same as the previous case. The isothermal conditions are considered where all the wall surfaces are at the same temperature. The whole annulus is modeled and the resulting mesh contains approximately 1.2 million cells. Different from above, the turbulence effects need to be included.

The two principle parameters for this flow is the Rossby number $R o=U / \Omega a$ and the axial Reynolds number $R e_{z}=2 U a / v$, where $U$ is the bulk average velocity of the axial 


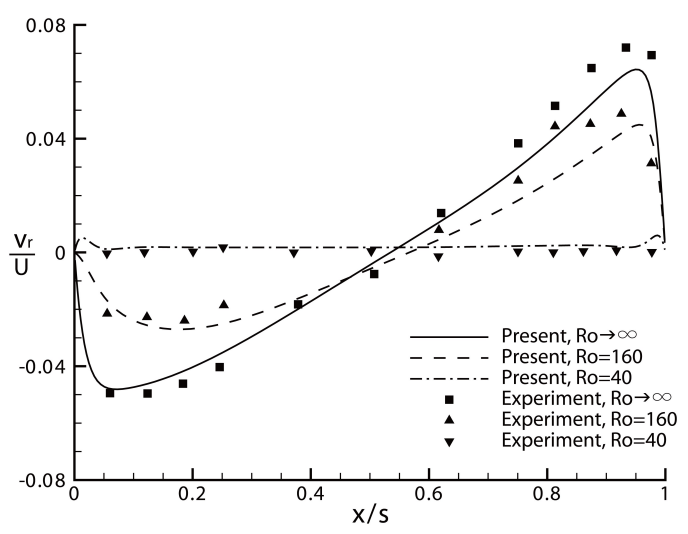

Figure 8: The axial variations of $v_{r} / U$ at $r / b=0.8$ for $R e_{z}=8 \times 10^{4}$ and $R o=\infty, 160$ and 40 .

throughflow. Fig. 7 shows the in-plane streamlines for $R e_{z}=8 \times 10^{4}$ and $R o=\infty, 160$, 80 and 40 . When there is no rotation $(R o=\infty)$, a strong symmetric toroidal vortex is generated inside the stationary cavity. For lower rotational speed $(R o=160)$, no obvious change is found in the flow behavior except the appearance of a weak vortex near the upstream disk. With further increase in rotational speed $(R o=80$ and 40$)$, the toroidal vortex is gradually suppressed and the fluid tends to rotate "purely" (the radial velocity is very small) over a wider region. For the current selected conditions, the flow fields are all in stable states showing excellent symmetries as seen in the figure. However, it should be noted that when the rotational speed is increased above certain values, the vortex breakdown could occur, leading to a number of characteristic flow modes which are probably related with instabilities. This issue is currently beyond the scope of this paper and leaves room for our follow-up study.

Fig. 8 shows the axial variations of the radial velocity $v_{r}$ at the radial position $r / b=0.8$ for $R e_{z}=8 \times 10^{4}$ and $R o=\infty, 160$ and 40. It is seen that the fluid moves inward near the upstream disk while moves outward near the downstream disk, which is consistent with the toroidal vortex shown in Fig. 7. With the increase of the rotational speed from zero to a higher value, the radial velocity is significantly decreased. For $R o=\infty$ and 160 , the computed results are in good agreements with the experimental data. The reasons for the underestimate near the downstream disk are probably because of the inconsistent setup with that in the real experiment and the error introduced by the turbulence model. For $R o=40$, both the numerical and experimental results show that the radial flow is suppressed to an extremely low level.

Fig. 9 further shows the radial variations of the tangential velocity $v_{\phi}$ at mid-axial plane for $R e_{z}=4 \times 10^{4}$ and $R o=160,80$ and 40. The agreements between the computed and experimental results are good, although the peak values at lower rotational speeds are slightly over-predicted. It is found that at higher $r / b$, the value of $v_{\phi} / \Omega r$ tends to 


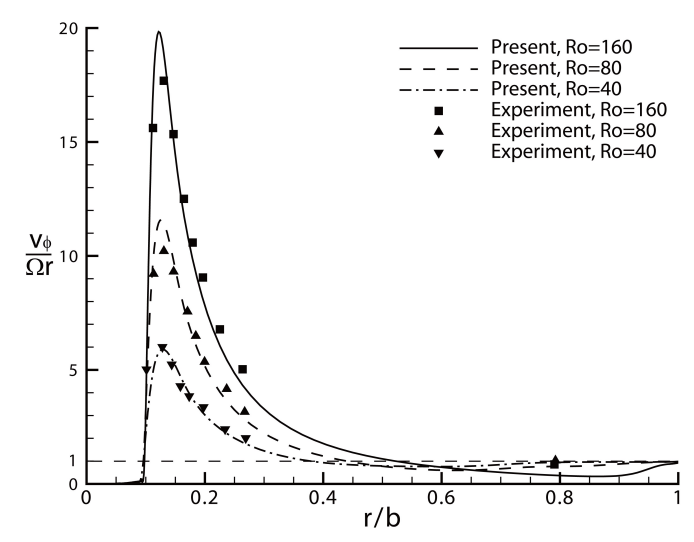

Figure 9: The radial variations of $v_{\phi} / \Omega r$ at mid-axial plane for $R e_{z}=4 \times 10^{4}$ and $R o=160,80$ and 40 .

unity, indicating that the flow is in near solid body rotation at outer part of the cavity. With the increase of the rotational speed, the peak value of $v_{\phi} / \Omega r$ decreases and the area of near-solid body rotation becomes larger, again confirming the flow patterns referred above.

The validity of the present method for incompressible rotating flows has been verified through the first two cases. Due to the regularity of the used mesh, the proposed new way and the regular way for calculating the spatial derivatives of $f_{0}$ and $g$ lead to almost the same results. In the following, two more complex and practical applications are considered.

Case 3: Low-speed centrifugal compressor.

This case is about the NASA low-speed subsonic centrifugal compressor, which was designed to investigate the essential flow physics of the flow field. A complete description of the experiment and the detailed measured data can be found in [29]. Fig. 10 shows a schematic diagram of the compressor, which contains a backswept impeller followed by a vaneless diffuser. The basic specifications of the compressor are shown in Table 1. The computational domain is characterized by a long twisted flow passage of high curvature and low aspect ratio, and the resulting mesh consists of approximately 0.8 million cells. At the inlet boundary, the distributions of the total pressure, total temperature and flow direction are specified, and the other variables are determined through the non-reflecting boundary condition and the isentropic condition. At the outlet boundary, the imposed static pressure has been adjusted to meet the target mass flow rate, and the other variables are extrapolated from the interior. As in the experiment, the hub wall is moving with the rotor blade, while the diffuser hub and the whole shroud are stationary. All the solid wall boundaries are assumed to be adiabatic. Periodic conditions are imposed along the circumferential boundaries. A design flow rate condition with the target mass flow rate $\dot{m}=30 \mathrm{~kg} / \mathrm{s}$ is chosen to be studied. 


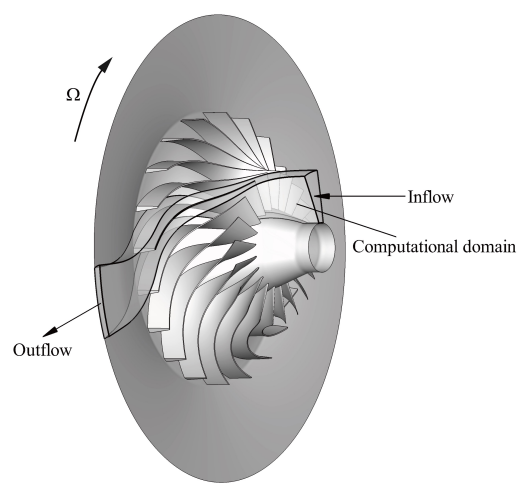

Figure 10: Schematic diagram of the centrifugal compressor.

Table 2 shows the comparison of the computed overall performance with the data from experiment and from reference [30]. Also presented are the results from the conventional N-S solver which uses the upwind Roe scheme for evaluation of inviscid fluxes. The adiabatic efficiency is computed based on the energy-averaged total pressure ratio and the mass-averaged total temperature ratio. It is found that the present method and the N-S solver give very similar results, both of which are in better agreements with the experimental data. Shown in Fig. 11 are comparisons of distributions of normalized reduced pressure $P_{r} / P_{r e f}$ at $5 \%, 49 \%, 79 \%$ and $98 \%$ spanwise sections from hub, respec-

Table 1: Basic specifications of the low-speed subsonic centrifugal compressor.

\begin{tabular}{||l|c|c||}
\hline Number of impeller blades & 20 \\
\hline \multirow{2}{*}{ Tip diameter $(\mathrm{m})$} & Inlet & 0.870 \\
\cline { 2 - 3 } & \multicolumn{1}{|c|}{ Exit } & 1.524 \\
\hline \multirow{2}{*}{ Blade height $(\mathrm{m})$} & Inlet & 0.218 \\
\cline { 2 - 3 } & Exit & 0.141 \\
\hline Backsweep angle $(\mathrm{deg})$ & 55 \\
\hline Clearance between blade tip and shroud $(\mathrm{mm})$ & 2.54 \\
\hline Rotational speed $(\mathrm{rpm})$ & 1862 \\
\hline Design tip speed $(\mathrm{m} / \mathrm{s})$ & 153 \\
Design mass flow rate $(\mathrm{kg} / \mathrm{s})$ & 30 \\
\hline
\end{tabular}

Table 2: Comparison of overall compressor performance at the design condition.

\begin{tabular}{||c|c|c|c|c||}
\hline & Experiment & Reference [30] & N-S solver & Present \\
\hline Energy-averaged total pressure ratio & 1.141 & 1.1395 & 1.1404 & 1.1400 \\
\hline Mass-averaged total temperature ratio & 1.042 & 1.0404 & 1.0416 & 1.0415 \\
\hline Adiabatic efficiency & 0.922 & 0.9415 & 0.9190 & 0.9189 \\
\hline
\end{tabular}




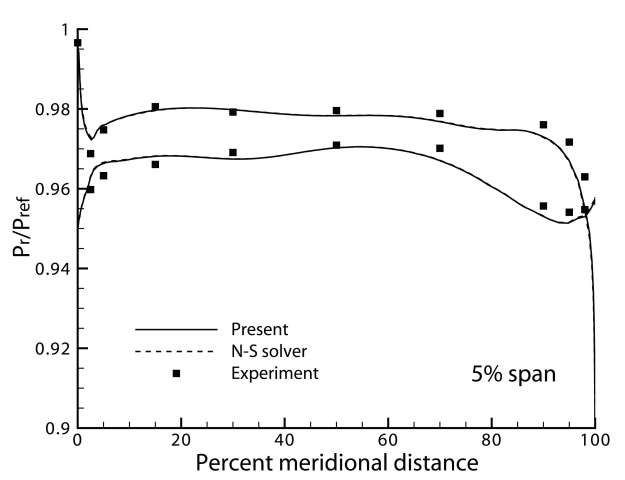

(a)

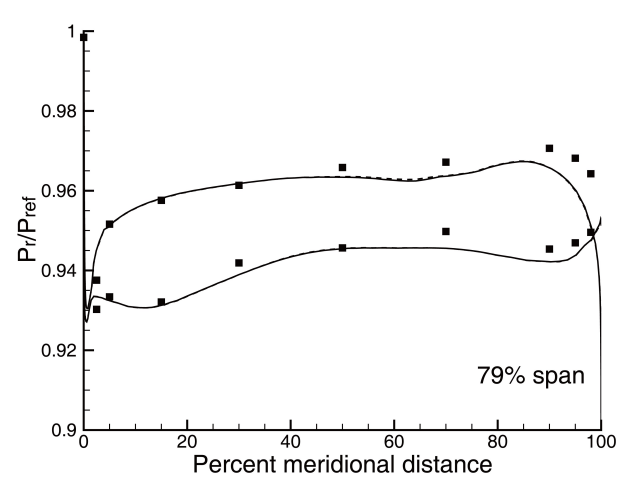

(c)

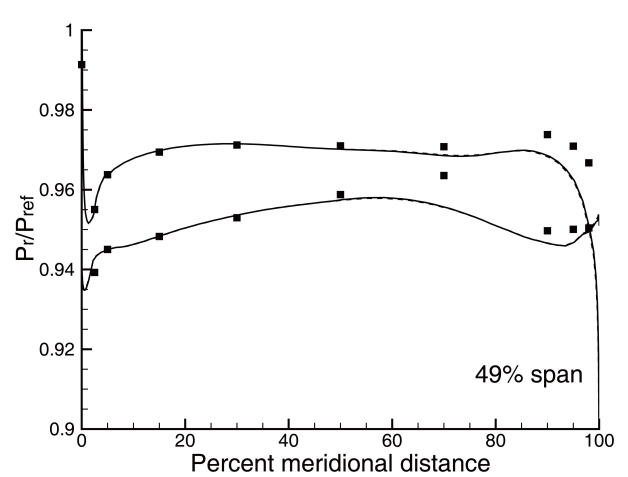

(b)

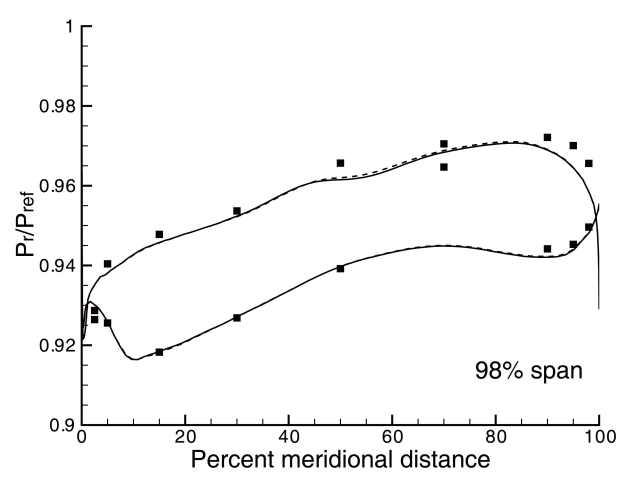

(d)

Figure 11: Comparisons of distributions of normalized reduced static pressure at different spanwise sections.

tively. The reduced pressure $P_{r}$ is defined as

$$
P_{r}=p\left[\frac{E^{*}}{E^{*}+\Omega^{2} r^{2} / 2}\right]^{\gamma /(\gamma-1)},
$$

where $p$ is the static pressure, $E^{*}$ is the relative total energy, and the reference pressure $P_{\text {ref }}$ is taken as the inlet total pressure. As seen in Fig. 11, by using both the present method and the N-S solver, overall good agreements are found over a wide range of blade surfaces. The discrepancies between predicted and experimental results can be similarly found in [30]. There are only slight differences between the numerical results at near blade tip and it seems that a better agreement with the experimental data is achieved. The good agreements can also be seen in Fig. 12, where the BGK scheme gives consistent isolines of reduced pressure on blade surfaces with those from experiment and reference. An obvious pressure gradient from hub to tip is observed in the majority of the flow channel, which is balanced with the wall curvature caused inertia and centrifugal forces and the rotation caused centrifugal and Coriolis forces. At near impeller outlet, the pres- 


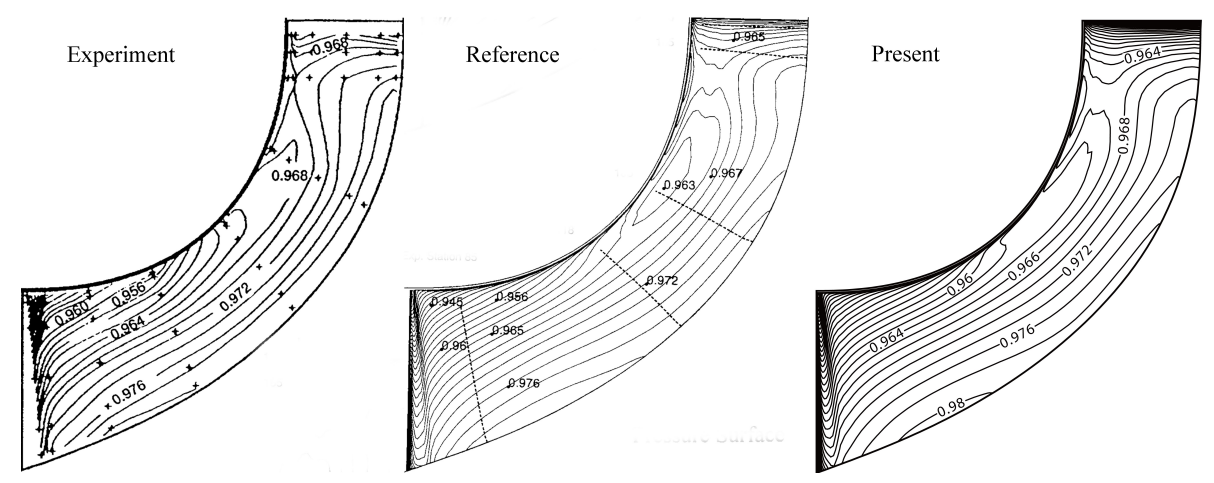

(a) Pressure surface

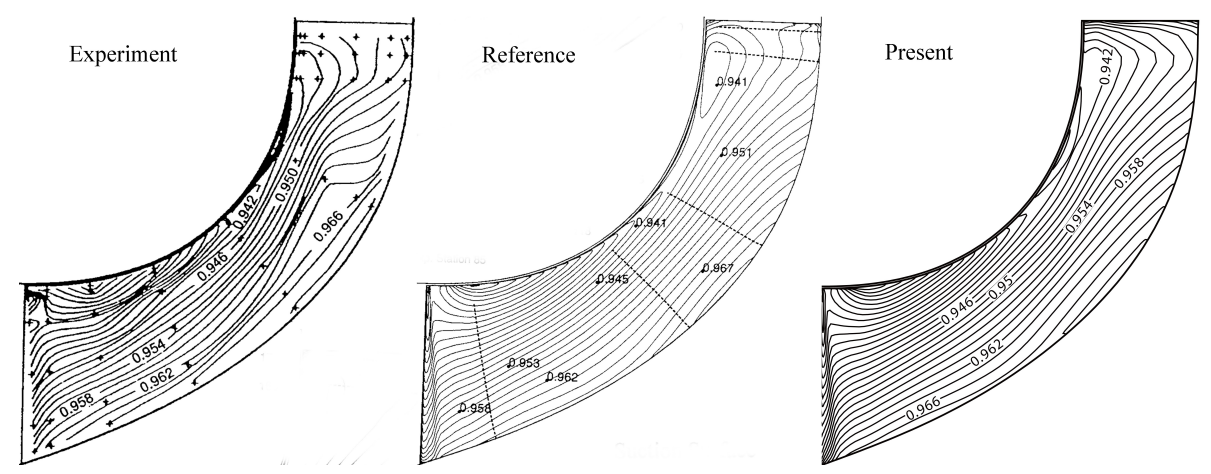

(b) Suction surface

Figure 12: Comparisons of isolines of normalized reduced static pressure on blade surfaces.

sure gradient gradually disappears and an almost uniform distribution is found. There are isoline loops on the pressure surface (PS), which is caused by the tip vortex shifting away from the shroud/PS corner. However, the predicted loop area seems slightly smaller than the other two ones. As for the suction surface (SS), the pressure gradient is higher than that on the pressure surface, and the predicted isolines are closer to the experimental results as compared with those from reference.

Because a full matching mesh (point-to-point mesh) is employed on periodic boundaries and the real tip clearance is modeled, there inevitably appear some grid cells of degrading qualities in such a narrow and long-twisted passage. For this case, when the original way to calculate the derivatives of $f$ and $g$ is used in the BGK scheme, the convergence difficulty could be encountered. However, by using the current approach, we successfully simulate the viscous flows in this practical rotating machinery and it is shown that the accuracy is comparable to that of the N-S solver.

Case 4: Transonic axial-flow fan rotor.

The last case presents the simulation of the transonic flow in a low-aspect-ratio axial- 


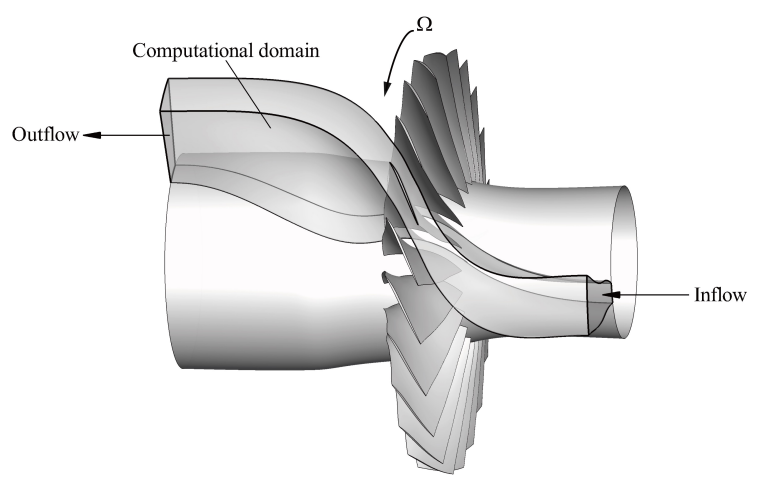

Figure 13: Schematic diagram of the transonic axial-flow fan rotor.

flow fan rotor, Rotor67, which was designed and experimentally analyzed at the NASA Leiws Research Center. Detailed experimental data and the rotor geometry can be referred to [31]. A schematic diagram of Rotor67 is shown in Fig. 13, and the basic specifications of the rotor are given in Table 3. As seen in Fig. 13, only a single flow passage is modeled due to the use of the periodic boundary conditions. The inlet boundary conditions are imposed in the same way as in the previous case. At the outlet boundary, the static pressure is specified at hub location and a radial equilibrium equation is solved to obtain the pressure distribution. The other variables are obtained through extrapolation from the interior. All the computations are performed on a fine mesh composed of about 1.6 million cells.

Fig. 14 first shows comparisons of the computed overall performance with the experimental results, including the variations of total pressure ratio $P t$ and the adiabatic efficiency $\eta$ with nondimensional mass flow rate. Also presented are the numerical re-

Table 3: Basic specifications of the transonic axial-flow fan rotor, Rotor67.

\begin{tabular}{|c|c|c|}
\hline \multicolumn{2}{|l|}{ Number of rotor blades } & 22 \\
\hline \multirow{2}{*}{ Tip diameter (m) } & Inlet & 0.514 \\
\hline & Exit & 0.485 \\
\hline \multirow{2}{*}{$\mathrm{Hub} /$ tip radius ratio } & Inlet & 0.375 \\
\hline & Exit & 0.478 \\
\hline \multicolumn{2}{|c|}{ Clearance between blade tip and shroud (mm) } & 1.016 \\
\hline \multicolumn{2}{|l|}{ Rotational speed (rpm) } & 16043 \\
\hline \multicolumn{2}{|l|}{ Rotor tip speed (m/s) } & 429 \\
\hline \multicolumn{2}{|c|}{ Inlet tip relative Mach number } & 1.38 \\
\hline \multicolumn{2}{|c|}{ Design mass flow rate $(\mathrm{kg} / \mathrm{s})$} & 33.15 \\
\hline \multicolumn{2}{|l|}{ Design pressure ratio } & 1.63 \\
\hline
\end{tabular}



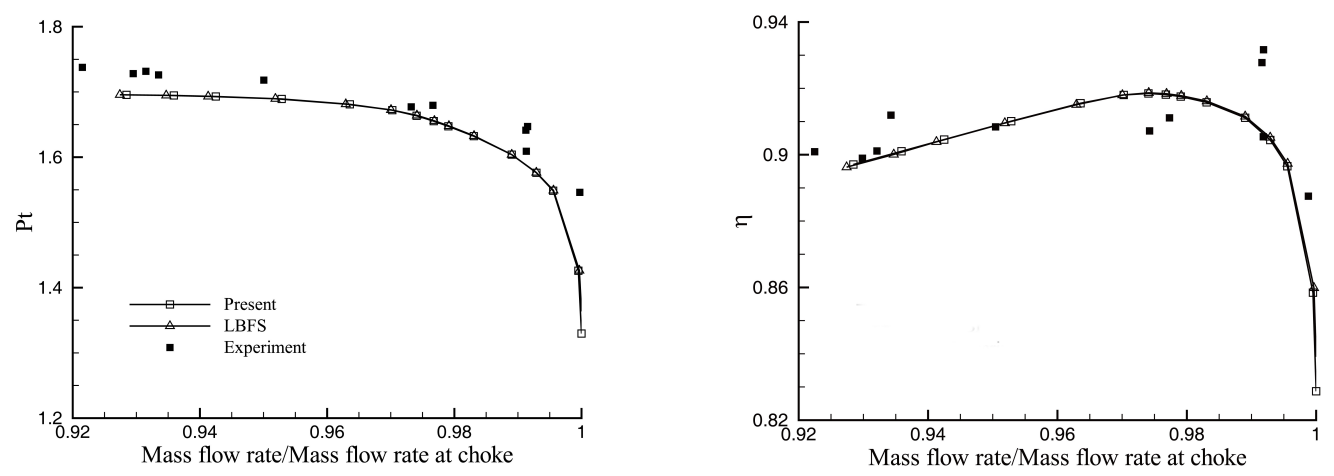

Figure 14: Comparisons of overall performance at design speed.

sults from a recently-developed lattice Boltzmann flux solver (LBFS) [20]. The predicted mass flow rates at choke by using the present method and the LBFS are $34.6469 \mathrm{~kg} / \mathrm{s}$ and $34.6534 \mathrm{~kg} / \mathrm{s}$, respectively, while the experimental value is $34.96 \mathrm{~kg} / \mathrm{s}$. The differences are both less than $1 \%$. It is seen from Fig. 14 that no apparent differences are found between the numerical results. The predicted rotor performance in general agrees well with the experimental data, although it is slightly under-predicted.

To further explore the capability of the present method for capturing flow details, a near-peak-efficiency condition which is consistent with the experimental setup is selected to be studied. Fig. 15 shows comparisons of the spanwise distributions of energyaveraged pressure ratio $P t$, mass-averaged temperature ratio $T t$ and area-averaged flow angle. Excellent agreements are found between the numerical results from the present method and the LBFS, despite of slight discrepancies at near endwall region where viscous effects dominate. The reason is probably because in the LBFS, only inviscid fluxes are reconstructed from the solution of the lattice Boltzmann equation while the viscous fluxes are calculated simply by central averaging. However, in the current BGK scheme, the invisicd and viscous fluxes are evaluated simultaneously as a whole. It is also seen from the figure that the predicted temperature ratio shows a favorable agreement with the experimental data but the pressure ratio is overall underestimated, which explains the under-prediction of overall performance shown in Fig. 14. The over-prediction of the flow angle can be similarly observed in [32].

Still for this near-peak-efficiency condition, Fig. 16 shows comparisons of the isolines of relative Mach number at 30\%, 70\% and 90\% span from hub, respectively. The computed contours in general agree well with those from the experiment. At 30\% span, the flow is entirely subsonic over the passage except for a small supersonic area near the leading edge. At $70 \%$ span, an obvious bow shock attached to the leading edge appears and impinges on the location of about $55 \%$ chord of the suction side of the adjacent blade. After the bow shock, there is also a weak passage shock. Basically, they do not greatly interfere with each other. At $90 \%$ span (near tip), a typical form of the $\lambda$-structure is 

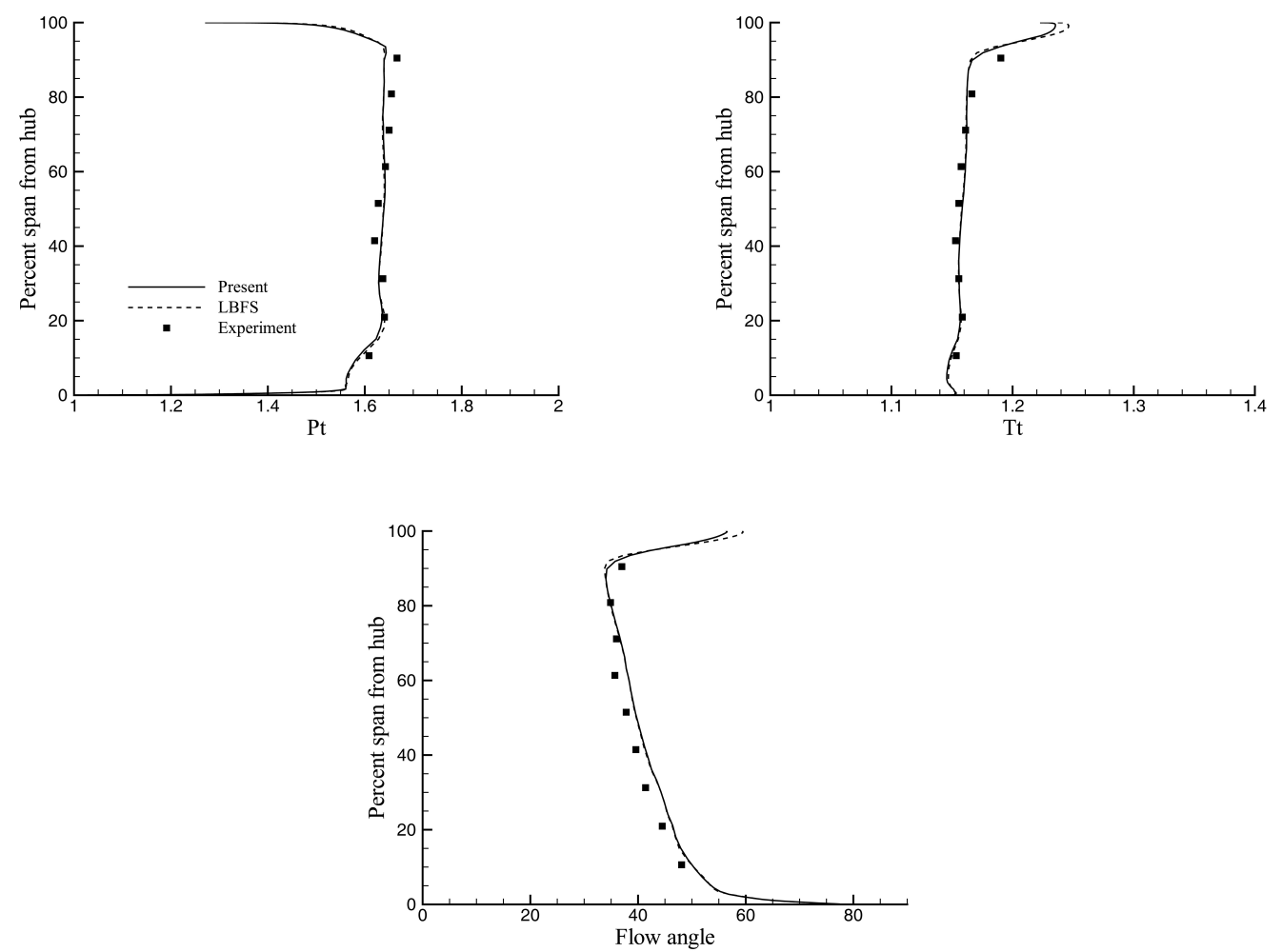

Figure 15: Comparisons of spanwise distributions of pressure ratio $\mathrm{Pt}$, temperature ratio $\mathrm{Tt}$ and flow angle for the near-peak-efficiency condition.

found as a result of the combined effects of the boundary layer, the bow shock and the passage shock. The flow over the passage is almost entirely supersonic. With the increase of the spanwise location and the resulted inflow velocity, the bow shock impinges on the location closer to the trailing edge of the adjacent blade surface, and the intensity of the passage shock becomes higher.

For the same reason demonstrated in the previous case, when the original way to calculate the derivatives of $f$ and $g$ is used without extra fixes, unreasonable values of the derivatives are first obtained at several cell interfaces. Then the influence of them would gradually spread to the whole flow field, causing floating point errors.

\section{Conclusions}

In this paper, aiming at simulation of viscous flows in rotating machinery, a threedimensional gas-kinetic BGK scheme is presented. The key point for developing the current method is to construct an appropriate BGK model for the N-S equations in a ro- 

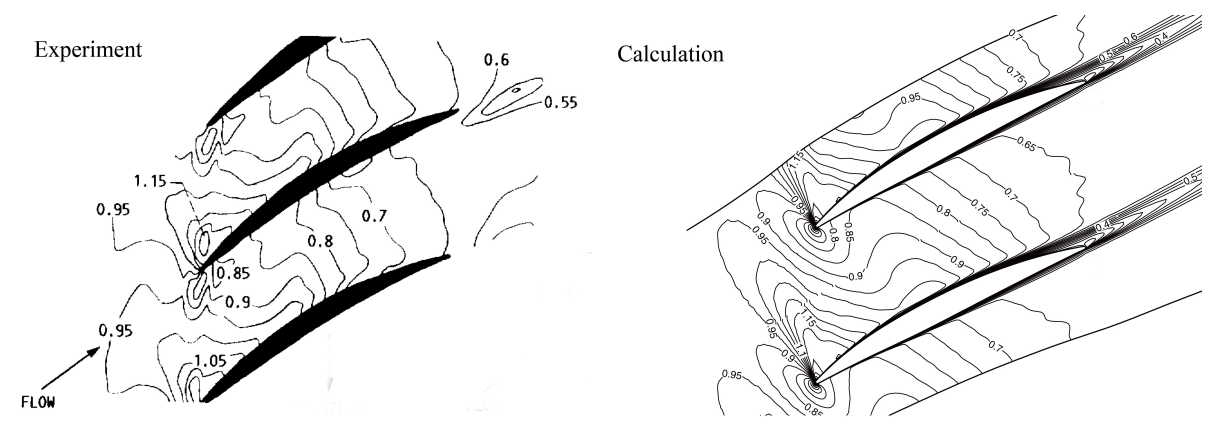

(a) $30 \%$ span
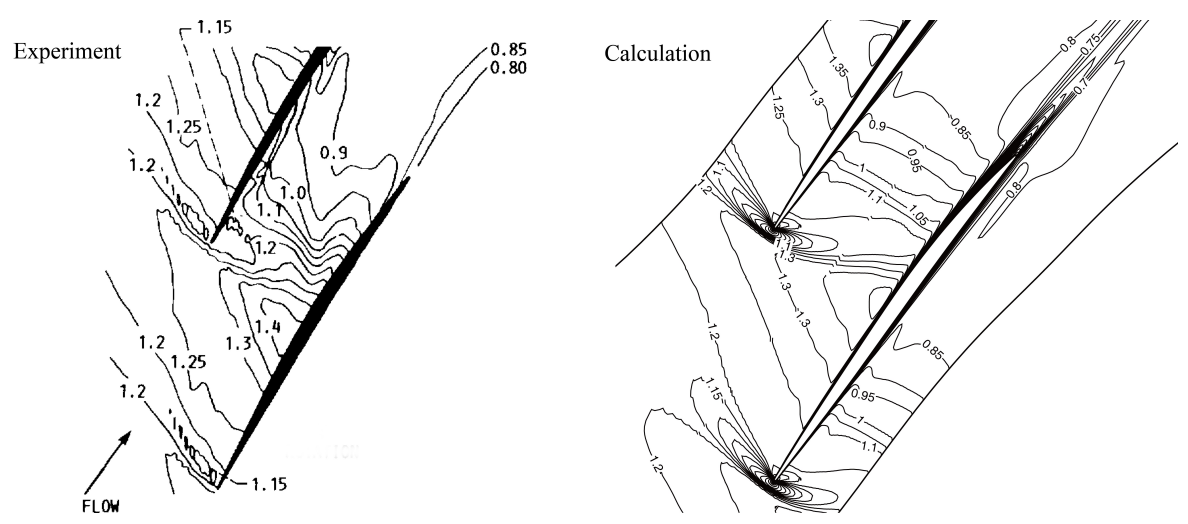

(b) $70 \%$ span
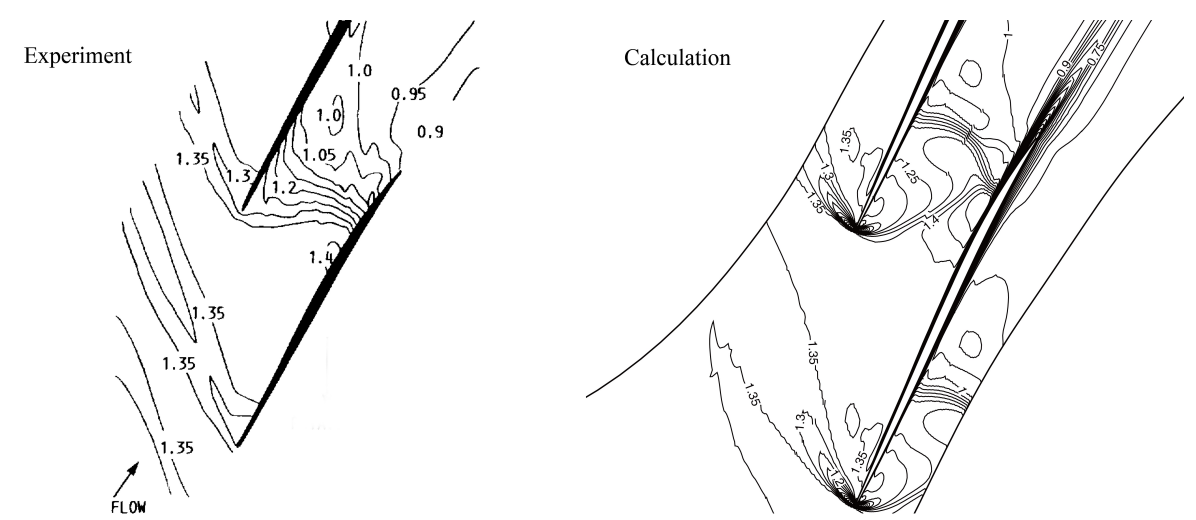

(c) $90 \%$ span

Figure 16: Comparisons of isolines of relative Mach number at different spanwise locations for the near-peakefficiency condition.

tating frame of reference. In order to include the non-inertial effects into the BGK model, a specifically-designed acceleration term is introduced. Under the finite-volume frame- 
work, the numerical fluxes at each cell interface are evaluated by reconstructing the solution of the modified Boltzmann equation, and subsequently the flow filed can be solved. To improve the modeling capability of the BGK scheme in practical rotating fluid applications where some distorted mesh may exist, we also propose to employ an improved approach to calculate the derivatives of gas distribution function and its equilibrium state. For validation, several tests cases are investigated. The computed results show that the developed method can be well applied to typical rotating flows with favorable accuracy and robustness. Through this work, the application scope of the BGK-type method is also extended.

\section{Appendix: Expressions of coefficients $a_{\alpha}, b_{\alpha}, c_{\alpha}, A_{\alpha},(\alpha=1 \sim 5)$ by using the chain rule}

The expression of equilibrium state is repeated here

$$
g=\rho\left(\frac{\lambda}{\pi}\right)^{\frac{N+3}{2}} e^{-\lambda\left(\left(\xi_{x}-u\right)^{2}+\left(\xi_{y}-v\right)^{2}+\left(\xi_{z}-w\right)^{2}+\zeta^{2}\right)} .
$$

Take the slope

$$
a=\frac{\partial g}{g \partial x}
$$

as an example, by using the chain rule for the above Maxwellian distribution function $g$, it can be expanded as follows

$$
a=\frac{\partial g}{g \partial x}=\frac{\partial g}{\partial \rho} \frac{\partial \rho}{\partial x}+\frac{\partial g}{\partial u} \frac{\partial u}{\partial x}+\frac{\partial g}{\partial v} \frac{\partial v}{\partial x}+\frac{\partial g}{\partial w} \frac{\partial w}{\partial x}+\frac{\partial g}{\partial \lambda} \frac{\partial \lambda}{\partial x}
$$

According to Section 2.2, $a$ can also be written as a linear combination of the collision invariants $\varphi_{\alpha},(\alpha=1 \sim 5)$, i.e.,

$$
a=a_{\alpha} \varphi_{\alpha}=a_{1}+a_{2} \xi_{x}+a_{3} \xi_{y}+a_{4} \xi_{z}+\frac{1}{2} a_{5}\left(\xi_{x}^{2}+\xi_{y}^{2}+\xi_{z}^{2}+\zeta^{2}\right)
$$

Comparing the above two equations, we directly get

$$
\begin{aligned}
& a_{1}=\frac{\partial \rho}{\rho \partial x}-2 \lambda\left(u \frac{\partial u}{\partial x}+v \frac{\partial v}{\partial x}+w \frac{\partial w}{\partial x}\right)+\left(\frac{N+3}{2 \lambda}-u^{2}-v^{2}-w^{2}\right) \frac{\partial \lambda}{\partial x} \\
& a_{2}=2\left(u \frac{\partial \lambda}{\partial x}+\lambda \frac{\partial u}{\partial x}\right) \\
& a_{3}=2\left(v \frac{\partial \lambda}{\partial x}+\lambda \frac{\partial v}{\partial x}\right) \\
& a_{4}=2\left(w \frac{\partial \lambda}{\partial x}+\lambda \frac{\partial w}{\partial x}\right) \\
& a_{5}=-2 \frac{\partial \lambda}{\partial x}
\end{aligned}
$$


As for the other coefficients $b_{\alpha}, c_{\alpha}$ and $A_{\alpha}$, they can be written in the same form as $a_{\alpha}$ except that the derivative symbol $\partial / \partial x$ in the above expressions is changed to $\partial / \partial y, \partial / \partial z$ and $\partial / \partial t$, respectively.

\section{Acknowledgements}

This work has been supported by the National Natural Science Foundation of China (Grant No. 11372135) and the National Basic Research Program of China ("973" Project) (Grant No. 2014CB046200).

\section{References}

[1] P. R. N. CHILDS, Chapter 1: Introduction to Rotating Flow, in: Rotating Flow, ButterworthHeinemann, Oxford, 2011, pp. 1-15.

[2] K. XU, Gas-kinetic schemes for unsteady compressible flow simulations, Lecture Series-van Kareman Institute for Fluid Dynamics, 03 (1998).

[3] E. F. TORO, Methods for Multi-Dimensional PDEs, in: E. F. Toro (Ed.) Riemann Solvers and Numerical Methods for Fluid Dynamics: A Practical Introduction, Springer Berlin Heidelberg, Berlin, Heidelberg, 2009, pp. 543-584.

[4] K. H. PRendergast AND K. XU, Numerical hydrodynamics from gas-kinetic theory, J. Comput. Phys., 109 (1993), pp. 53-66.

[5] K. XU, A gas-kinetic BGK scheme for the Navier-Stokes equations and its connection with artificial dissipation and Godunov method, J. Comput. Phys., 171 (2001), pp. 289-335.

[6] Z. L. GUO, H. W. LIU, L.S. LUO AND K. XU, A comparative study of the LBE and GKS methods for 2D near incompressible laminar flows, J. Comput. Phys., 227 (2008), pp. 4955-4976.

[7] Q. B. LI, S. FU AND K. XU, Application of gas-kinetic scheme with kinetic boundary conditions in hypersonic flow, AIAA J., 43 (2005), pp. 2170-2176.

[8] Y. S. LIAN AND K. XU, A gas-kinetic scheme for multimaterial flows and its application in chemical reactions, J. Comput. Phys., 163 (2000), pp. 349-375.

[9] D. ZHOU, Z. L. LU, T. Q. GUO AND L. M. YANG, Development of circular function-based gas-kinetic scheme (CGKS) on moving grids for unsteady flows through oscillating cascades, Int. J. Numer. Methods Fluids, (2017).

[10] K. XU AND J. C. HuANG, A unified gas-kinetic scheme for continuum and rarefied flows, J. Comput. Phys., 229 (2010), pp. 7747-7764.

[11] K. XU, A gas-kinetic scheme for the Euler equations with heat transfer, SIAM J. Sci. Comput., 20 (1999), pp. 1317-1335.

[12] D. ZHOU, Z. L. LU AND T. Q. GUO, Development of a moving reference frame-based gas-kinetic $B G K$ scheme for viscous flows around arbitrarily moving bodies, J. Comput. Phys., (under review), (2018).

[13] G. MAY, B. SRINIVASAN AND A. JAMESON, An improved gas-kinetic BGK finite-volume method for three-dimensional transonic flow, J. Comput. Phys., 220 (2007), pp. 856-878.

[14] Q. B. LI, K. XU AND S. FU, A high-order gas-kinetic Navier-Stokes flow solver, J. Comput. Phys., 229 (2010), pp. 6715-6731.

[15] L. TANG, Progress in gas-kinetic upwind schemes for the solution of Euler/Navier-Stokes equations I: Overview, Comput. Fluids, 56 (2012), pp. 39-48. 
[16] C. Q. JIN AND K. XU, A unified moving grid gas-kinetic method in Eulerian space for viscous flow computation, J. Comput. Phys., 222 (2007), pp. 155-175.

[17] K. XU, M. L. MAO AND L. TANG, A multidimensional gas-kinetic BGK scheme for hypersonic viscous flow, J. Computt. Phys., 203 (2005), pp. 405-421.

[18] C. T. TIAN, K. XU, K. L. Chan AND L. C. DENG, A three-dimensional multidimensional gaskinetic scheme for the Navier-Stokes equations under gravitational fields, J. Comput. Phys., 226 (2007), pp. 2003-2027.

[19] J. LUO AND K. XU, A high-order multidimensional gas-kinetic scheme for hydrodynamic equations, Sci. China Tech. Sci., 56 (2013), pp. 2370-2384.

[20] D. ZHOU, Z. L. LU AND T. Q. GUO, A rotating reference frame-based lattice Boltzmann flux solver for simulation of turbomachinery flows, Int. J. Numer. Methods Fluids, 83 (2017), pp. 561582.

[21] S. W. XIOng, C. W. ZhOng, C. S. Zhuo, K. Li, X. P. Chen And J. CaO, Numerical simulation of compressible turbulent flow via improved gas-kinetic BGK scheme, Int. J. Numer. Methods Fluids, 67 (2011), pp. 1833-1847.

[22] D. X. PAN, C. W. ZhONG, J. Li AND C. S. ZhuO, A gas-kinetic scheme for the simulation of turbulent flows on unstructured meshes, Int. J. Numer. Methods Fluids, 82 (2016), pp. 748-769.

[23] P. Spalart AND S. Allmaras, A one-equation turbulence model for aerodynamic flows, in: 30th Aerospace Sciences Meeting and Exhibit, American Institute of Aeronautics and Astronautics, 1992.

[24] D. ZHOU, Z. L. LU AND T. Q. GUO, Improvement of computational efficiency of circular functionbased gas kinetic scheme by using Jacobian-free Newton-Krylov method, Comput. Fluids, 161 (2018), pp. 121-135.

[25] D. CHAE, C. KIM AND O.-H. RHO, Development of an improved gas-kinetic BGK scheme for inviscid and viscous flows, J. Comput. Phys., 158 (2000), pp. 1-27.

[26] G. D. van Albada, B. VAn Leer And W. W. Roberts, A Comparative Study of Computational Methods in Cosmic Gas Dynamics, in: M. Y. Hussaini, B. van Leer, J. Van Rosendale (Eds.) Upwind and High-Resolution Schemes, Springer Berlin Heidelberg, Berlin, Heidelberg, 1997, pp. 95-103.

[27] J. M. OWen, J. R. Pincombe AND R. H. ROgers, Source-sink flow inside a rotating cylindrical cavity, J. Fluid Mech., 155 (1985), pp. 233-265.

[28] P. R. FArthing, C. A. Long, J. M. OWen And J. R. Pincombe, Rotating cavity with axial throughflow of cooling air: heat transfer, J. Turbomachinery, 114 (1992), pp. 229-236.

[29] M. D. HathawaY, R. M. Chriss, A. J. STRAZISAR AND J. R. WOOD, Laser anemometer measurements of the three-dimensional rotor flow field in the NASA low-speed centrifugal compressor, (1995).

[30] S. KANG AND C. HiRSCH, Numerical investigation of the three-dimensional flow in NASA lowspeed centrifugal compressor impeller, in: International Symposium on Experimental and Computational Aerothermodynamics of Internal Flows, 1999.

[31] A. J. STRAZiSAR, J. R. WOOD, M. D. HATHAWAY AND K. L. Suder, Laser anemometer measurements in a transonic axial-flow fan rotor, (1989).

[32] T. ARImA, T. SONODA, M. SHIROTORI, A. TAMURA AND K. KIKUCHI, A numerical investigation of transonic axial compressor rotor flow using a Low-Reynolds-Number $k-\varepsilon$ turbulence model, J. Turbomachinery, 121 (1999), pp. 44-58. 